\title{
Perovskite solid solutions with multiferroic morphotropic phase boundaries and property enhancement
}

\author{
M. Algueró* ${ }^{\S}$, H. Amorín*, C. M. Fernández-Posada*, O. Peña ${ }^{\dagger}$, \\ P. Ramos*, E. Vila* and A. Castro* \\ *Instituto de Ciencia de Materiales de Madrid \\ CSIC, Cantoblanco, 28049 Madrid, Spain \\ $\dagger^{\prime}$ Institut des Sciences Chimiques de Rennes \\ Associé au CNRS (UMR 6226) \\ Université de Rennes 1, Rennes 35042, France \\ tDepartamento de Electrónica \\ Universidad de Alcalá, 28871 Alcalá de Henares, Spain \\ \$malguero@icmm.csic.es
}

Received 17 December 2015; Revised 17 February 2016; Accepted 14 March 2016; Published 5 May 2016

\begin{abstract}
Recently, large phase-change magnetoelectric response has been anticipated by a first-principles investigation of phases in the $\mathrm{BiFeO}_{3}-\mathrm{BiCoO}_{3}$ perovskite binary system, associated with the existence of a discontinuous morphotropic phase boundary (MPB) between multiferroic polymorphs of rhombohedral and tetragonal symmetries. This might be a general property of multiferroic phase instabilities, and a novel promising approach for room temperature magnetoelectricity. We review here our current investigations on the identification and study of additional material systems, alternative to $\mathrm{BiFeO}_{3}-\mathrm{BiCoO}_{3}$ that has only been obtained by high pressure synthesis. Three systems, whose phase diagrams were, in principle, liable to show multiferroic MPBs have been addressed: the $\mathrm{BiMnO}_{3}-\mathrm{PbTiO}_{3}$ and $\mathrm{BiFeO}_{3}-\mathrm{PbTiO}_{3}$ binary systems, and the $\mathrm{BiFeO}_{3}-\mathrm{BiMnO}_{3}-\mathrm{PbTiO}_{3}$ ternary one. A comprehensive study of multiferroism across different solid solutions was carried out based on electrical and magnetic characterizations, complemented with mechanical and electromechanical measurements. An in-depth structural analysis was also accomplished when necessary.
\end{abstract}

Keywords: Multiferroics; magnetoelectrics; perovskite solid solutions; morphotrophic phase boundary; ceramic technologies.

\section{Introduction}

The search of single-phase multiferroics with large room temperature (RT) magnetoelectric response has concentrated and increasing activity during the last 10 years, ${ }^{1-3}$ for they are an enabling material technology for a wide range of potentially disruptive related technologies that would make use of the ability of controlling electrical polarization with the magnetic field, and magnetization with an electric one. Among the different applications proposed, electrically tunable magnetic microwave components and spintronic devices, high sensitivity magnetic sensors with RT operation, and above all, the-long time chased electrical-writing magneticreading random access memory stand out. ${ }^{4-6}$

Although the first multiferroic compounds were described as early as in $1966,{ }^{7}$ these materials became highly topical at the dawn of the 21 st century after a number of reports of large magnetoelectric effects, basically changes of permittivity and reorientation of polarization, even direction reversal, under high magnetic fields in several magnetic (or spin induced) ferroelectrics. ${ }^{8,9}$ These are improper ferroelectrics, in which a small $\left(<<1 \mu \mathrm{C} \mathrm{cm}^{-2}\right)$ spontaneous polarization develops as a byproduct of a magnetic transition, often involving complex incommensurate antiferromagnetic states like sinusoidal or spiral spin configurations. ${ }^{10}$ First reported materials were orthorhombic terbium manganites like $\mathrm{TbMnO}_{3}$ and $\mathrm{TbMn}_{2} \mathrm{O}_{5}$ with ordering temperatures around $40 \mathrm{~K}$, which developed a ferroelectric state at low temperature and successive magnetic transitions involving spin reorientation. Large magnetoelectric effects were phase-change responses associated with the shift of the antiferromagnetic state instability with the magnetic field. ${ }^{8-10}$ Following works concentrated on finding systems that showed similar phenomenology but higher magnetic ordering temperatures, among which $\mathrm{CuO}$ at $230 \mathrm{~K},{ }^{11}$ and $\mathrm{Sr}_{3} \mathrm{Co}_{4} \mathrm{Fe}_{24} \mathrm{O}_{41}$ at $670 \mathrm{~K}$ deserved being highlighted. ${ }^{12}$ However, a comparable electrical control of magnetization has not been achieved. Therefore, and though research goes on covering an ever wide range of manganites, ferrites, chromites and cobaltites, ${ }^{13}$ alternative material concepts need to be (and are being) investigated.

A second set of materials under extensive research are $\mathrm{ABO}_{3}$ perovskites in which multiferroism is chemically

This is an Open Access article published by World Scientific Publishing Company. It is distributed under the terms of the Creative Commons Attribution 4.0 (CC-BY) License. Further distribution of this work is permitted, provided the original work is properly cited. 
engineered by placing ferroelectrically and magnetically active cations in the $A$ - and $B$-site, respectively. $\mathrm{Pb}^{2+}$ or $\mathrm{Bi}^{3+}$ with stereochemically active $6 s^{2}$ electron lone pairs are usually selected for the A-site, while magnetically active $\mathrm{Mn}^{3+}$, $\mathrm{Fe}^{3+}, \mathrm{Co}^{3+}, \mathrm{Ni}^{3+}$ and $\mathrm{Cr}^{3+}$ are located at the $\mathrm{B}$-site, like for the ferroelectric antiferromagnets $\mathrm{Pb}\left(\mathrm{Fe}_{1 / 2} \mathrm{Nb}_{1 / 2}\right) \mathrm{O}_{3}$ and $\mathrm{BiFeO}_{3}{ }^{14}$ This A-site-based ferroelectricity would result from a different mechanism to that responsible of the ferroic order in model ferroelectric compounds like $\mathrm{BaTiO}_{3}$ and $\mathrm{PbTiO}_{3}$, for which the hybridization between the Ti $3 \mathrm{~d}$ and $\mathrm{O}$ 2 p electronic states and thus, covalency plays a major role. ${ }^{15}$ Recall that an intrinsic contradiction has been pointed out to exist between B-site-based ferroelectricity, which involves a transition metal in $a \mathrm{~d}^{\circ}$ configuration, and magnetism that requires the same metals with partially filled (with an odd number of electrons) d levels. ${ }^{16}$

Indeed, $\mathrm{BiFeO}_{3}$ stands out as the most topical multiferroic at present, not only for it is one of the rare compounds that show independent but coupled electrical and magnetic orders at RT, ${ }^{17,18}$ but for its magnetic and ferroelectric domain configurations are related, which enables the reorientation of the antiferromagnetic domains following the electrical switching of the ferroelectric/ferroelastic ones. ${ }^{19,20}$ A large effort is being put in order to tailor down its high RT conductivity, while enhancing its weak ferromagnetism. ${ }^{21}$ Besides, this material is a basic component of multiferroic film epitaxial heterostructures under extensive investigation for magnetoelectricity. ${ }^{22}$

Recently, partially reversible ferroelectric domain reorientations under magnetic fields have been described for $(1-x) \mathrm{Pb}\left(\mathrm{Zr}_{0.53} \mathrm{Ti}_{0.47}\right) \mathrm{O}_{3}-x \mathrm{~Pb}\left(\mathrm{Fe}_{1 / 2} \mathrm{Ta}_{1 / 2}\right) \mathrm{O}_{3}$ with $x=0.4$ at RT. ${ }^{23}$ This is an example out of a number of perovskite solid solutions between ferroelectric phases and relaxor compounds containing magnetically active cations which show weak ferromagnetism in coexistence with a range of polar states, and different magnetoelectric coupling phenomena. ${ }^{24}$ Spin clustering is thought to be responsible for the ferromagnetic component, while elastic interactions between the magnetic nanoregions and the ferroelectric matrix would cause the magnetoelectric effects.

Also involving perovskite solid solutions, strong phasechange magnetoelectric response has been anticipated by a first-principles investigation of phases in the $\mathrm{BiFeO}_{3}-\mathrm{BiCoO}_{3}$ binary system, associated with the existence of a discontinuous morphotropic phase boundary (MPB) between multiferroic polymorphs of rhombohedral and tetragonal symmetries. ${ }^{25}$ Mechanism would be the discrete rotation of the magnetization easy axis following that of the spontaneous polarization, during the electric field induced transformation from one ferroelectric (multiferroic) polymorphic phase to the other.

Furthermore, a recent experimental study reported the existence of a monoclinic $\mathrm{Cm}$ phase between the rhombohedral and tetragonal polymorphs in the $\mathrm{BiFeO}_{3}-\mathrm{BiCoO}_{3}$ system, and polarization rotation within this phase with temperature and composition. ${ }^{26}$ The phase diagram would be then analogous to that described for high sensitivity piezoelectric $\mathrm{Pb}(\mathrm{Zr}, \mathrm{Ti}) \mathrm{O}_{3}$, which shows lattice transverse softening and very high polarizability and piezoelectric response by the mechanism of continuous polarization rotation. ${ }^{27,28}$ The finding of the same intermediate monoclinic phase in the multiferroic system raises the possibility of also obtaining an enhanced magnetoelectric response by the same mechanism.

Indeed, these distinctive magnetoelectric effects might be a general property of multiferroic MPBs; this understood as a boundary in the phase diagram separating two perovskite polymorphic phases both multiferroic, but with different symmetry and thus, spontaneous polarization (and coupled magnetization easy axis) along different crystal directions, and a novel promising approach for obtaining RT magnetoelectricity. However, the in-depth exploration of this approach requires the identification and study of additional material systems. A serious issue when addressing this task is the low thermodynamical stability of most of the suitable candidates, anticipated by their low perovskite tolerance factor. $\mathrm{BiFeO}_{3}-\mathrm{BiCoO}_{3}$, for instance, has only been obtained by high pressure synthesis. We review in this paper our current investigations on the identification of such material systems, in which mechanosynthesis in high energy planetary mills is playing a major role as an alternative to high pressure synthesis for obtaining these oxides. ${ }^{29}$

\section{The $\mathrm{BiMnO}_{3}-\mathrm{PbTiO}_{3}$ Binary System}

$\mathrm{BiMnO}_{3}$ is one of the rare ferromagnetic insulators with an ordering temperature of $100 \mathrm{~K},{ }^{30}$ which has also been claimed to be ferroelectric. Ferroelectricity was initially proposed after structural studies that showed this oxide to have a heavily distorted perovskite structure with monoclinic symmetry and $C 2$ space group, ${ }^{31}$ and the independent reports of ferroelectric hysteresis loops ${ }^{32}$ and second harmonic generation. ${ }^{33}$ Contemporary first-principles calculations indicated the off-center distortions to be driven by the stereochemically active $\mathrm{Bi}^{3+}$ lone pair. ${ }^{34}$ Indeed $\mathrm{BiMnO}_{3}$ was considered until recently as a prototype of multiferroics, in which independent electric and magnetic ferroic orders are obtained by placing ferroelectrically and magnetically active cations at the A-site and $\mathrm{B}$-site of the $\mathrm{ABO}_{3}$ perovskite. Therefore, it seemed a suitable perovskite edge member to be combined with the $\mathrm{PbTiO}_{3}$ tetragonal perovskite, in order to obtain a multiferroic MPB.

There was a single previous report of the $\mathrm{xBiMnO}_{3}-(1-x)$ $\mathrm{PbTiO}_{3}$ perovskite solid solutions series when we started working on the system. ${ }^{35}$ These authors succeeded in obtaining perovskite single phases up to an $x$ value of 0.7 by conventional solid-state synthesis, and described a tetragonal $P 4 \mathrm{~mm}$ phase for $x$ below 0.4 , and a pseudocubic phase for $x$ values above this threshold. No phase coexistence was found for any composition that might indicate the existence of a 


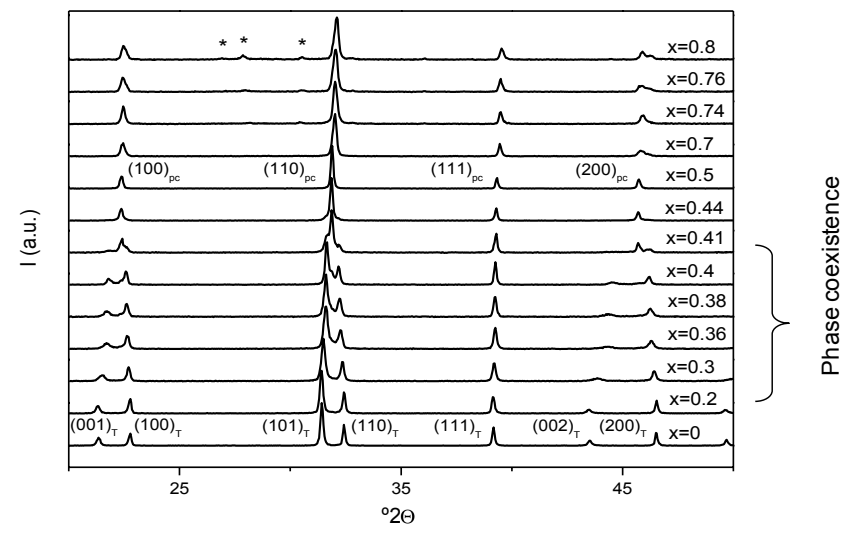

(a)

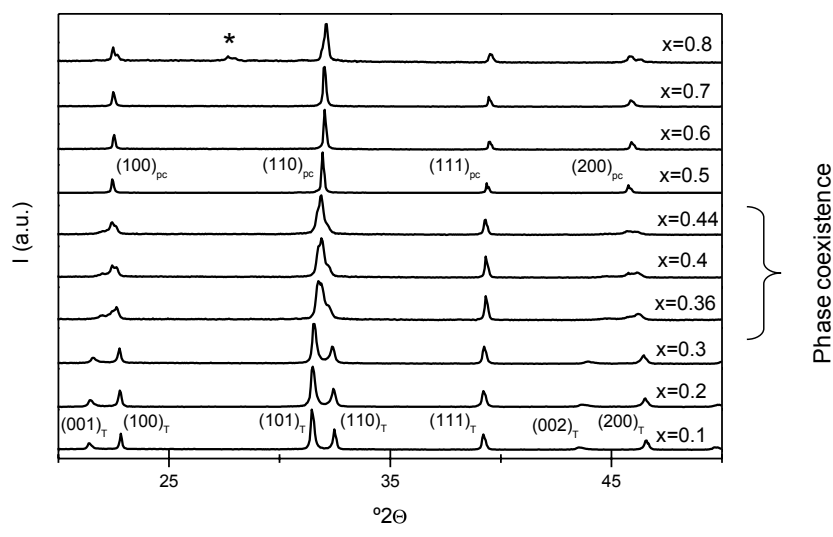

(b)

Fig. 1. XRD patterns of highly crystalline $x \mathrm{BiMnO}_{3}-(1-x) \mathrm{PbTiO}_{3}$ powders obtained by (a) wet chemistry (adapted with permission from Ref. 37. Copyright (2010) American Chemical Society), and (b) mechanosynthesis (adapted from Ref. 38, Copyright () 2012 WILEY-VCH Verlag GmbH \& Co. KGaA, Weinheim). Peaks associated with the coexisting tetragonal (T) and pseudocubic (pc) polymorphic phases are indicated with Miller indices. *Second phases.

MPB, so the structural evolution was proposed to be associated with the disruption of the long range ferroelectric order with increasing $x$, and the appearance of a relaxor state. ${ }^{36}$

However, our own investigations clearly showed the existence of a compositional range between $x=0.36$ and 0.44 , where coexistence of two perovskite polymorphic phases was found, which suggested the targeted MPB to exist. Consistent results were obtained on two independent set of powdered samples synthesized by a nonconventional wet chemistry approach ${ }^{37}$ and by mechanosynthesis, ${ }^{38}$ respectively. High chemical homogeneity is provided by these alternative advanced synthesis techniques, which is thought to have been the key to uncovering the MPB. In summary, two perovskite polymorphic phases are present in the system: (1) a tetragonal $P 4 \mathrm{~mm}$ phase for $0 \leq x<0.36$, and (2) an apparently cubic, yet we will see later that this is not the case, phase for $x>0.44$, while a region where these tetragonal and pseudocubic phases coexist is found for intermediate compositions, namely $0.36 \leq x \leq 0.44$. This is illustrated in Fig. 1, where X-ray diffraction (XRD) patterns of the two sets of powders are provided.

Note the presence of traces of second phases for $x>0.74$ in the case of the powders synthesized by wet chemistry. This method involved a final thermal treatment at $1273 \mathrm{~K}$ in the case of materials with $x$ up to 0.6 , which was decreased down to $1223 \mathrm{~K}$ above this value and up to 0.8 . No perovskite phases were obtained above this composition. Indeed, the magnetic characterization clearly showed the presence of a magnetic impurity for $x$ values as low as $x=0.2$.

Traces of the same second phase were found for the material with $x=0.8$ obtained by mechanosynthesis. Nanocrystalline powders result from the mechanochemical activation and thus, a thermal treatment at $1223 \mathrm{~K}$ was required to increase crystallinity for the structural characterization. The second phase appears after this thermal treatment, for its magnetic signature was not found in the nanocrystalline powders that were perovskite single phase according to both XRD and selected area electron diffraction (SAED) in transmission electron microscopy (TEM). It is remarkable that the technique allowed perovskite phases to be obtained all across the binary system beyond 0.8 , including $\mathrm{BiMnO}_{3}$ $(x=1)$, which had only been obtained before by high pressure synthesis. However, these nanocrystalline perovskites were unstable under heating, and thermal decomposition was triggered at a temperature that decreased when $x$ increased, such as it was $1173 \mathrm{~K}$ for $x=0.5,{ }^{38}$ and $673 \mathrm{~K}$ for $x=1 .^{39}$

Perovskite structure evolution across the system. Perovskite lattice parameters were obtained as a function of composition for the two polymorphs, and are shown in Fig. 2. Consistent trends were found in the two sets of powders, so as the tetragonal distortion, $c / a$, maintained a constant value around 1.07 up to $x=0.2$, above which it started decreasing on approaching the phase coexistence region down to $\sim 1.03$ for $x=0.44$. Slightly smaller $c$ parameters and thus, tetragonal distortions were obtained in the case of the mechanosynthesized powders. Perovskite cell volume is also shown in the figure for the two polymorphs. Note the small volume difference between the tetragonal and pseudocubic phases within the coexistence region, which seems to suggest a discontinuous MPB. This difference is noticeable in the case of the materials synthesized by wet chemistry, but not for those obtained by mechanosynthesis. Like the differences in $c / a$, this is most probably related to the remnant microcrystalline strain; that is, a high level of perovskite disorder characteristic of the mechanosynthesis, which is not fully removed during subsequent thermal treatments, and that is thought to play a major role in the stabilization of the low tolerance factor pervoskites. ${ }^{29,40}$

The actual symmetry of the pseudocubic phase was studied on highly crystalline samples of $x=0.5$ and 0.6 by 

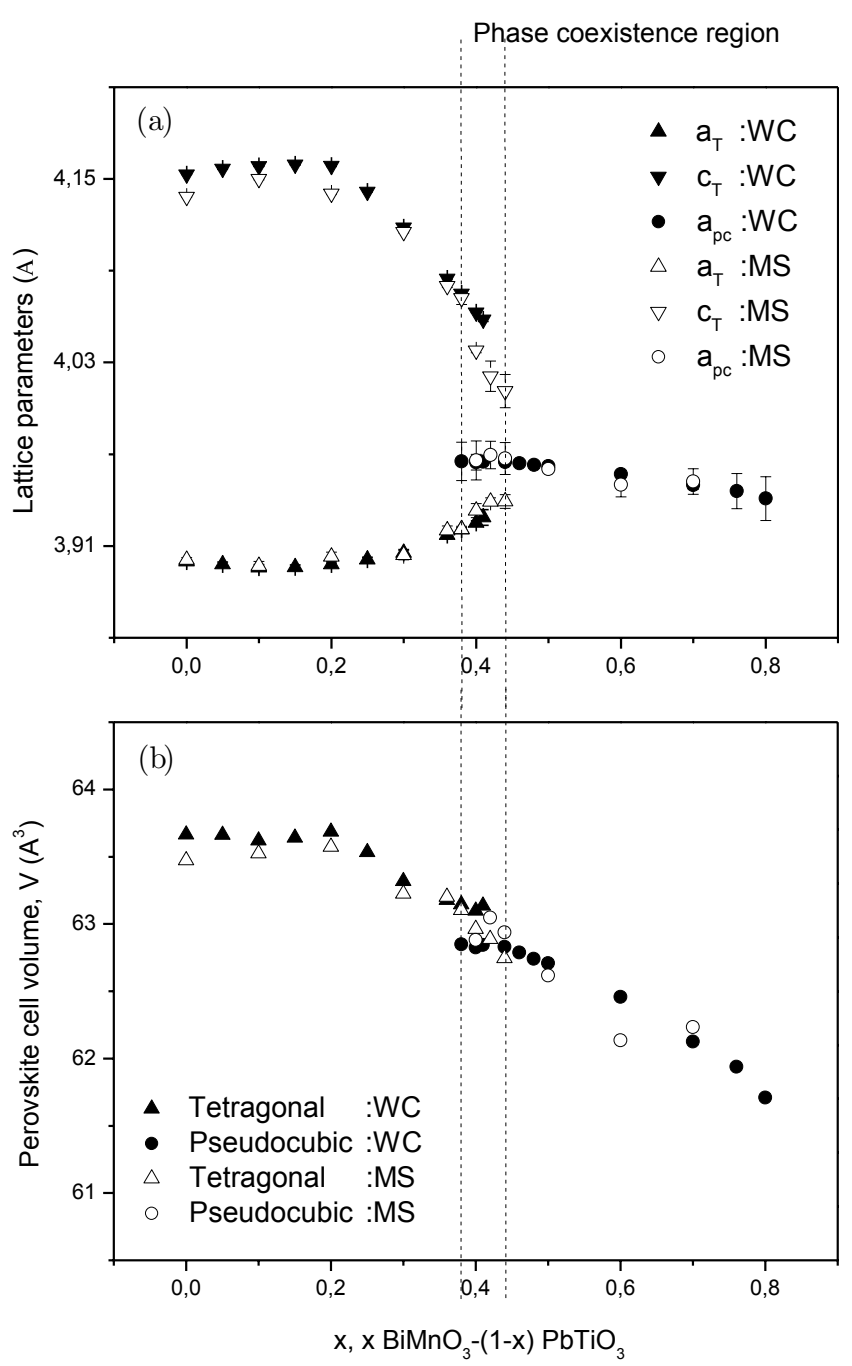

Fig. 2. (a) Perovskite lattice parameters of the tetragonal $\left(a_{T}\right.$ and $\left.c_{T}\right)$ and pseudocubic $\left(a_{p c}\right)$ polymorphic phases across the $x \mathrm{BiMnO}_{3}-$ $(1-x) \mathrm{PbTiO}_{3}$ solid solution series for the powders obtained by either wet chemistry: WC (from Ref. 37) or mechanosynthesis: MS (from Ref. 38), and (b) evolution of the perovskite cell volume.

precession electron diffraction, and found to be orthorhombic Pnma. Details of the space group determination can be found elsewhere. ${ }^{38}$ This is the same crystal structure of the high temperature phase of $\mathrm{BiMnO}_{3}$, which transforms into the monoclinic one at $770 \mathrm{~K}^{41,42}$ The monoclinic to orthorhombic transformation can be also induced by high pressures, ${ }^{43}$ and by oxygen hyperstoichiometry, ${ }^{44}$ as well as by the simultaneous substitution of $\mathrm{Pb}^{2+}$ for $\mathrm{Bi}^{3+}$ and $\mathrm{Ti}^{4+}$ for $\mathrm{Mn}^{3+}$ as shown here. This might be an example of chemical pressure effects, like those described in the $\mathrm{Pb}(\mathrm{Zr}, \mathrm{Ti}) \mathrm{O}_{3}$ system that have been proposed to be responsible of the appearance of the MPB. ${ }^{45}$ Multiferroic $\mathrm{BiFeO}_{3}$ also presents a high temperature orthorrombic Pnma phase that transforms into the rhombohedral $R 3 c$ phase at $\sim 1100 \mathrm{~K}^{18}$ Note that $P n m a$ is a centrosymmetrical group and thus, ferroelectricity is not allowed. Indeed the Pnma to $C 2$ transition at $770 \mathrm{~K}$ has been proposed to be the ferroelectric transition of $\mathrm{BiMnO}_{3}$, likewise the Pnma to $R 3 c$ transition of $\mathrm{BiFeO}_{3}$. It seems contradictory, however, that the substitutions of ferroelectrically active $\mathrm{Pb}^{2+}$ and $\mathrm{Ti}^{4+}$ for $\mathrm{Bi}^{3+}$ and $\mathrm{Mn}^{3+}$ result in the transformation of a polar phase into a nonpolar one. We will come back to this point in Sec. 3 .

Magnetism across the system. Though $\mathrm{BiMnO}_{3}$ is distinctively ferromagnetic, the increasing $\mathrm{Mn}^{3+}$ dilution with decreasing $x$ along the system readily transforms it into a canted antiferromagnetic with a Néel temperature that decreases with $x$, from $22 \mathrm{~K}$ at $x=0.9$ to $7 \mathrm{~K}$ for $x=0.3$. A weak paramagnetic state has been described below this temperature.

Details of the magnetic characterization and all results can be found somewhere else. ${ }^{37,38}$ We will focus here on analyzing possible differences between the magnetism of the two polymorphs within the coexistence region. Magnetization after zero field cooling (ZFC), measured during heating and subsequent field cooling (FC) between 2 and $120 \mathrm{~K}$ are shown in Fig. 3 for the powders obtained by mechanosynthesis. Curves for $x=0.3$ (tetragonal at RT), $x=0.4$ (phase coexistence) and $x=0.5$ (orthorhombic) are provided. No qualitative changes are found across the structural evolution, and all materials show a distinctive upturn of magnetization below $50 \mathrm{~K}$, along with well-defined maxima in the ZFC curve that indicate the Néel temperature, and a significant irreversibility or separation of the ZFC and FC curves, associated with the occurrence of spin canting and of weak ferromagnetism within the antiferromagnetic state. ${ }^{38}$ Actual changes are an expected increase of magnetization with $x$,

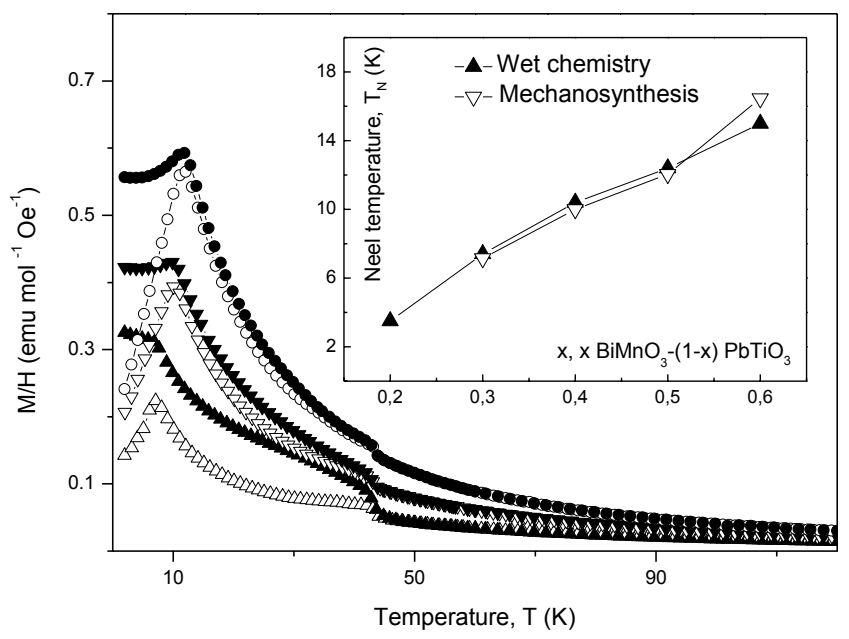

Fig. 3. Magnetization for the $x \mathrm{BiMnO}_{3}-(1-x) \mathrm{PbTiO}_{3}$ powders with $x=0.3(\triangle), 0.4(\nabla)$ and $0.5(O)$ obtained by mechanosynthesis (adapted from Ref. 38, Copyright (C) 2012 WILEY-VCH Verlag $\mathrm{GmbH} \& \mathrm{Co}$. KGaA, Weinheim). Measurements were carried out between 2 and $300 \mathrm{~K}$ after ZFC (open symbols) and during subsequent FC (full symbols) under an applied field of $100 \mathrm{Oe}$. The evolution of the Néel temperature across the solid solution series is provided in the inset, along with that obtained for powders synthesized by wet chemistry. 
i.e., with the concentration of $\mathrm{Mn}^{3+}$ cations, and shift of the Néel temperature. Consistent results were attained for the powders synthesized by wet chemistry. ${ }^{37}$

The shift of the Néel temperature with $x$ is illustrated in the inset of the figure for the two types of powder. No distinctive change of the slope of the $T_{N}$ versus $x$ curve is found across the phase coexistence, in spite of the described structural evolution. Recall that a small volume difference existed between the coexisting polymorphs, and so the $\mathrm{Mn}^{3+}-\mathrm{O}^{2-}-\mathrm{Mn}^{3+}$ superexchange interactions must be slightly modified.

Finally, note the presence of a magnetization step at $\sim 44$ $\mathrm{K}$ for all samples, associated with the previously discussed magnetic impurity.

Ferroelectricity across the system. The vanishing of ferroelectricity, anticipated by the perovskite structural evolution from a tetragonal $P 4 \mathrm{~mm}$ phase to an orthorrombic Pnma one, was independently confirmed by following the ferroelectric transition from $\mathrm{PbTiO}_{3}$ with increasing $x$. This was done by measuring the temperature dependence of a number of physical quantities, such as enthalpy changes, dielectric permittivity and Young's modulus that show distinctive signals and critical behavior at the transition.

The ferroelectric transition was initially followed by differential thermal analysis in the powdered samples synthesized by wet chemistry. ${ }^{37}$ The transition for $\mathrm{PbTiO}_{3}$ takes place at $\sim 765 \mathrm{~K}$ and is first order, so it has associated a distinctive reversible thermal signal readily identified in DTA. Analogous endothermic reversible effects that could be directly related to the transition were found for low $x$ values and up to $x=0.25$, so as the transition temperature, $T_{c}$, seemed to maintain a constant value around 770 and $760 \mathrm{~K}$ on heating and cooling, respectively, up to $x=0.2$, above which it started decreasing. However, the thermal signals were not observed above $x=0.25$, in spite of the tetragonal phase persisting up to $x=0.44{ }^{37}$ This indicates the presence of a tricritical point in the binary system; that is a composition at which the ferroelectric transition changes from first order to second order. This $x$ value seems to be between 0.25 and 0.30 for $x \mathrm{BiMnO}_{3}-(1-\mathrm{x}) \mathrm{PbTiO}_{3}$, and it is the composition at which the perovskite tolerance factor that continuously decreases with $x$ across the solid solution series takes a value of 1. Analogous tricritical points have been described for a number of perovskite systems, such as $\mathrm{Bi}\left(\mathrm{Mg}_{3 / 4} \mathrm{~W}_{1 / 4}\right) \mathrm{O}_{3}-$ $\mathrm{PbTiO}_{3}{ }^{46}$ Note also that this specific composition is that above which the tetragonal distortion starts a fast decrease towards the region of phase coexistence.

The ferroelectric transition was also followed by measuring the temperature dependence of the dielectric permittivity, after the processing of ceramics. In the case of materials processed by conventional sintering of the powders synthesized by wet chemistry, consistent transition temperatures were obtained up to $x=0.25$. However, conductivity was an issue above this value, and the dielectric anomaly associated with the transition vanished for higher values,

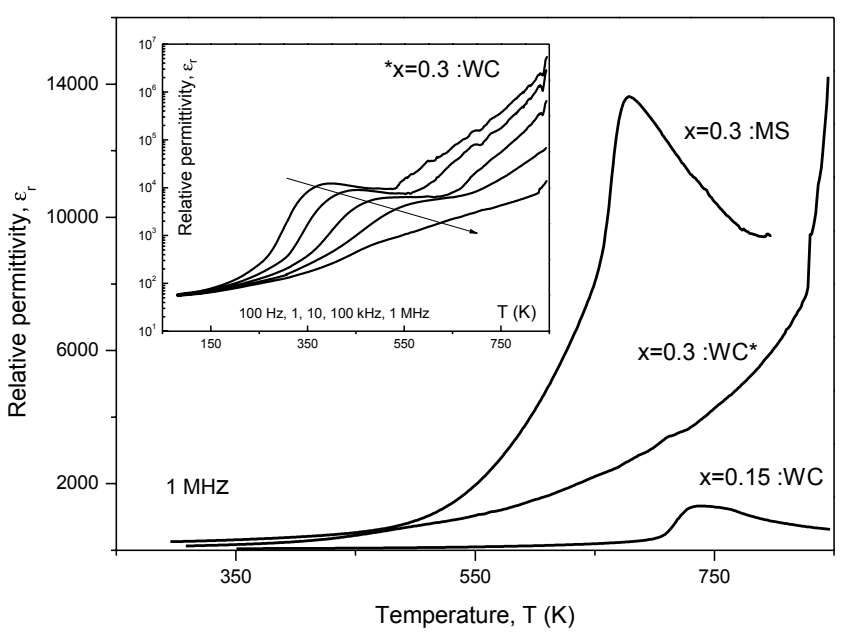

Fig. 4. Temperature dependence of the relative permittivity at 1 $\mathrm{MHz}$ of two $x \mathrm{BiMnO}_{3}-(1-x) \mathrm{PbTiO}_{3}$ ceramics with $x=0.15$ and $0.3^{*}$, processed by conventional sintering of powders obtained by wet chemistry: WC (from Ref. 37), and of a second material with $x=0.3$, this processed by hot pressing of the nanocrystalline powder obtained by mechanosynthesis: MS (from Ref. 47). *The frequency dispersion for this sample is provided in the inset.

basically buried in a strong high temperature dielectric relaxation and the conduction background (see inset of Fig. 4). Conductivity in this system is thought to be associated with the presence of an oxygen hyperstoichiometry, or rather of Mn vacancies and thus, $\mathrm{Mn}^{4+}$, by a mechanism of electron hopping between $\mathrm{Mn}^{3+}$ and $\mathrm{Mn}^{4+}{ }^{47}$ Conduction tailoring has been demonstrated by modifying synthesis and processing, and so microstructure, so as ceramics with $x=0.3$ processed by hot pressing of powders obtained by mechanosynthesis did show the dielectric anomaly at $678 \mathrm{~K},{ }^{47}$ as illustrated in Fig. 4.

Beyond $x=0.3$, the ferroelectric transition could only be followed by measuring the temperature dependence of the low frequency Young's modulus by dynamical mechanical analysis. This is a powerful means of studying phase transitions that has been widely applied to ferroelectrics complementing electrical measurements, especially useful when conduction becomes an issue like for $\mathrm{BiMnO}_{3}-\mathrm{PbTiO}_{3}{ }^{37,47}$ The technique has also been key to provide a description of the development of ferroelectric long range order in relaxor systems, ${ }^{48,49}$ and is also sensitive to the dynamics of the ferroelectric/ferroelastic domain walls. ${ }^{50}$

The temperature dependences of the dielectric permittivity and the Young's modulus across the ferroelectric transition of the ceramic material with $x=0.3$, processed by hot pressing of mechanosynthesized powders, are given in Fig. 5. Note the presence of a distinctive minimum in Young's modulus at the ferroelectric transition, readily identified by the characteristic dielectric anomaly. This is accompanied by a maximum in mechanical losses (not shown) that reflects the appearance of highly mobile ferroelectric/ferroelastic domains at the 


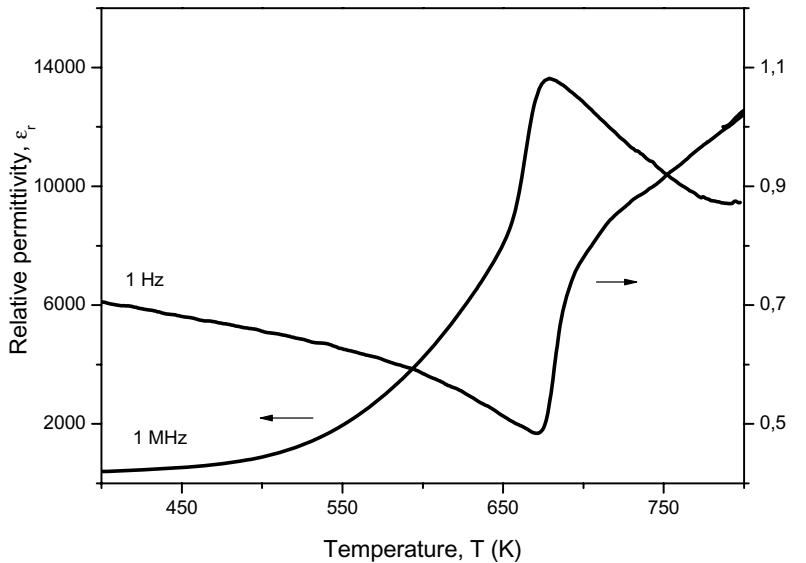

(a)

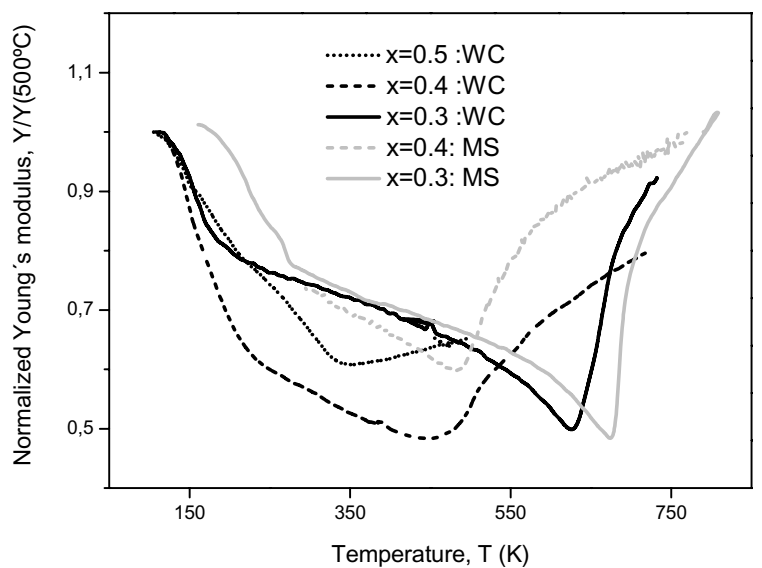

(b)

Fig. 5. (a) Temperature dependences of the relative permittivity and low frequency Young's modulus of one $x \mathrm{BiMnO}_{3}-(1-x) \mathrm{PbTiO}_{3}$ ceramic with $x=0.3$, processed by hot pressing of the nanocrystalline powder obtained by mechanosynthesis, across its ferroelectric transition, and (b) Temperature dependence of the Young's modulus of five $x \mathrm{BiMnO}_{3}-(1-x) \mathrm{PbTiO}_{3}$ ceramics with $x=0.3,0.4$ and 0.5 , processed by conventional sintering of powders obtained by wet chemistry: WC (from Ref. 37), and of two materials with $x=0.3$ and $x=0.4$, these ones processed by hot pressing of the nanocrystalline powder obtained by mechanosynthesis: MS (from Ref. 47 and unpublished, respectively).

transition; $90^{\circ}$ domains in the case of the tetragonal $P 4 \mathrm{~mm}$ phase.

The Young's modulus for ceramic materials with $x$ values up to 0.5 , processed from powders obtained by both wet chemistry and mechanosynthesis, are also given in the figure. The minima associated with the ferroelectric transition are easily identified in the curves of all materials with $x=0.3$ and 0.4 , so as the transition temperature further decreased down to $485 \mathrm{~K}$ in the latter case. However, an elastic anomaly that could be unambiguously associated with the transition was not observed for $x=0.5$.

Note also the presence of a low temperature mechanical effect consisting of a sharp increase of the Young's modulus for all materials, at a temperature that increases with $x$. This hardening has been also observed in rhombohedral $\mathrm{BiScO}_{3}-$ $\mathrm{PbTiO}_{3}$, and related to a low temperature phase transition. ${ }^{51}$ Its actual nature is not known, but it has clearly associated the disappearance, or at least freezing of the ferroelectric/ferroelastic domains.

All critical temperatures, whatever technique has been used to determine them, for the materials prepared by wet chemistry or mechanosynthesis are collected in Fig. 6, where the transition temperatures of the ceramic samples with $x=$ 0.2 and 0.3 , prepared by conventional means in Ref. 35 are included for completeness. A distinctive trend is obtained for the ferroelectric transition: initially, it maintains a constant value around $765 \mathrm{~K}$ up to $x=0.2$, composition at which the ferroelectric transition changes from first order to second order. Above this value, the transition temperature readily decreases down to a value of $485 \mathrm{~K}$ for $x=0.4$. Note that slightly higher $T_{c} \mathrm{~s}$ are obtained for the mechanosynthesized samples, yet this does not modify the trend. At the same time, the low temperature hardening takes place at an increasing temperature, so as it occurs at $330 \mathrm{~K}$ for $x=0.5$. This is above RT, at which an orthorhombic Pnma has been described. Therefore, one can conclude that the low temperature transition is a P4mm to Pnma transition, which involves the vanishing of ferroelectricity (and so of the improper ferroelasticity) and that it is shifted up with composition. Note that the Pnma phase appears at a temperature above the extrapolation of the $T_{C}$ curve for $x=0.5$. Therefore, a direct cubic Pm3m to orthorrombic Pnma can be assumed in this case.

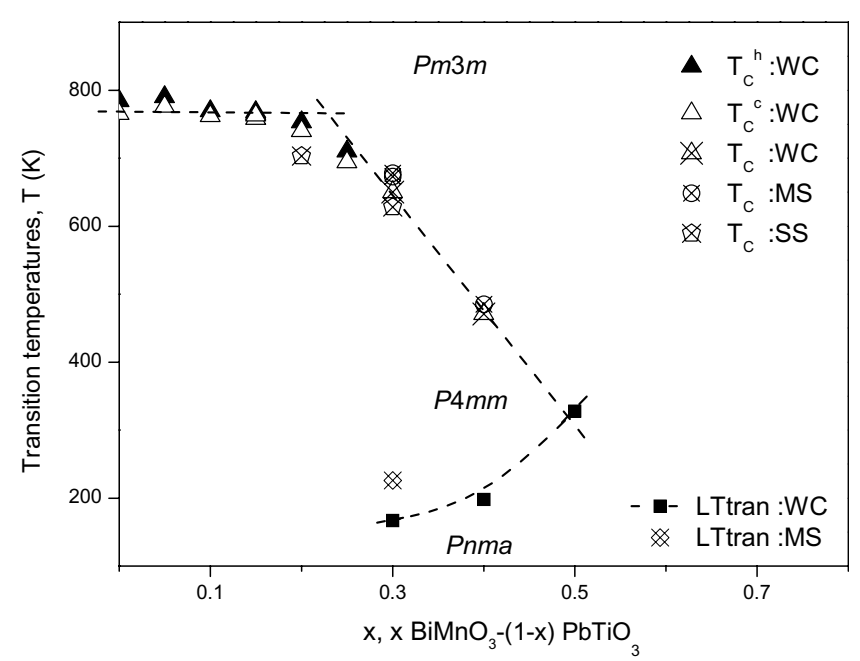

Fig. 6. Experimentally determined ferroelectric transition temperatures $\left(T_{C}^{h}\right.$ determined on heating, $T_{C}^{c}$ on cooling and $T_{C}$ no thermal hysteresis), and temperatures of the second polymorphic transition (LTtran) across the $x \mathrm{BiMnO}_{3}-(1-x) \mathrm{PbTiO}_{3}$ solid solution series for the samples obtained by conventional means: SS (from Ref. 35), wet chemistry: WC (from Ref. 37), and mechanosynthesis: MS (from Ref. 38). 
Indeed, this transition has been reported for $\mathrm{BiFeO}_{3}$ at high temperature. ${ }^{18}$ Recent high temperature XRD measurements have revealed that the coexisting polymorphs in powders with $x=0.4$ undergo independent structural transitions to a cubic phase on heating. This demonstrates the actual existence of a MPB in the system, though between a ferroelectric and a nonferroelectric phase, and bent at low temperatures towards $\mathrm{PbTiO}_{3}$.

Analysis of multiferroism.Summing up, antiferromagnetic order exists in the system for $x$ values as low as 0.3 that is also ferroelectric, but with an ordering temperature of $7 \mathrm{~K}$. This is well below the temperature at which the P4 $\mathrm{mm}$ to Pnma transition takes place, and ferroelectricity is lost. Therefore, and though ferroelectric and antiferromagnetic orders develop for $x=0.3$ and 0.4 , ferroelectricity vanishes before the magnetic one sets in, so they do not coexist at any temperature. Multiferroism then does not exist in the system.

Actually, note that magnetic ordering always takes place in orthorhombic phases, which explains the lack of discontinuities in the $T_{N}$ versus $x$ curve shown in Fig. 3. Recall that even if $x=0.3$ is tetragonal at RT, it transforms into the orthorhombic phase at $167 \mathrm{~K}$.

\section{Revisiting $\mathrm{BiMnO}_{3}$}

The vanishing of ferroelectricity in the $x \mathrm{BiMnO}_{3}-(1-x)$ $\mathrm{PbTiO}_{3}$ system for $x \geq 0.5$, which is when the orthorrombic Pnma phase develops, questions the actual ferroelectric nature of $\mathrm{BiMnO}_{3}$. Indeed, density functional calculations have shown that the ground state of this perovskite oxide is monoclinic $C 2 / c$, and thus centrosymmetric. ${ }^{52} \mathrm{BiMnO}_{3}$ would be then isostructural to $\mathrm{BiScO}_{3}$, and a comparative Rietveld study of neutron diffraction data seemed to suggest this to be the case. ${ }^{53}$ Subsequent additional neutron diffraction experiments confirmed this space group determination, ${ }^{44}$ but an independent report using SAED indicated the presence of long-ranged monoclinic $C 2$ order, in coexistence with short ranged $P 2$ or $P 2_{1}$ distortions, and thus, of a polar state. ${ }^{54}$ The length of the short range order seems to increase with the oxygen hyperstoichiometry, ${ }^{39}$ but a $P 2_{1} / c$ space group has been determined for $\mathrm{BiMnO}_{3.08} \cdot{ }^{44}$

Further insight into this issue can only be gained by a sound electrical characterization, which requires the processing of high quality ceramics. This is challenging for $\mathrm{BiMnO}_{3}$ that thermally decomposes into a mixture of $\mathrm{Bi}_{2} \mathrm{Mn}_{4} \mathrm{O}_{10}$, $\mathrm{Bi}_{12} \mathrm{MnO}_{20}$ and $\mathrm{MnO}_{2}$ above $673 \mathrm{~K}$. Note that $\mathrm{Bi}_{2} \mathrm{Mn}_{4} \mathrm{O}_{10}$ is ferromagnetic with a $T c$ of $42 \mathrm{~K}$, and most probably the magnetic impurity in Fig. 3.

We figured out the processing of dense ( $92 \%$ densification) ceramics of $\mathrm{BiMnO}_{3}$ by using spark plasma sintering and the nanocrystalline powders obtained by mechanosynthesis. ${ }^{39}$ Oxygen hyperstoichiometry was controlled by XPS and magnetic measurements, by comparing the Néel temperature of $80 \mathrm{~K}$ to previous reports on the evolution of the magnetism with stoichiometry. ${ }^{44}$ A composition of $\mathrm{BiMnO}_{3.04}$ was estimated, so monoclinic $C 2 / c$ phase can be assumed. ${ }^{39}$

A full electrical characterization was carried out. ${ }^{39}$ The temperature dependence of the dielectric permittivity is shown in Fig. 7. Samples thermally decomposed when heated above $673 \mathrm{~K}$, so the temperature of the monoclinic to orthorhombic transition at $770 \mathrm{~K}$ could not be reached. Results basically show up to three successive dielectric relaxations on heating from $77 \mathrm{~K}$, but no reversible nondispersive anomaly that could be associated with a phase transition. Note the relaxation peaks in the $375-475 \mathrm{~K}$ range that had been previously confused with the ferroelectric transition, for $\mathrm{BiMnO}_{3}$ shows a first structural phase transition between two

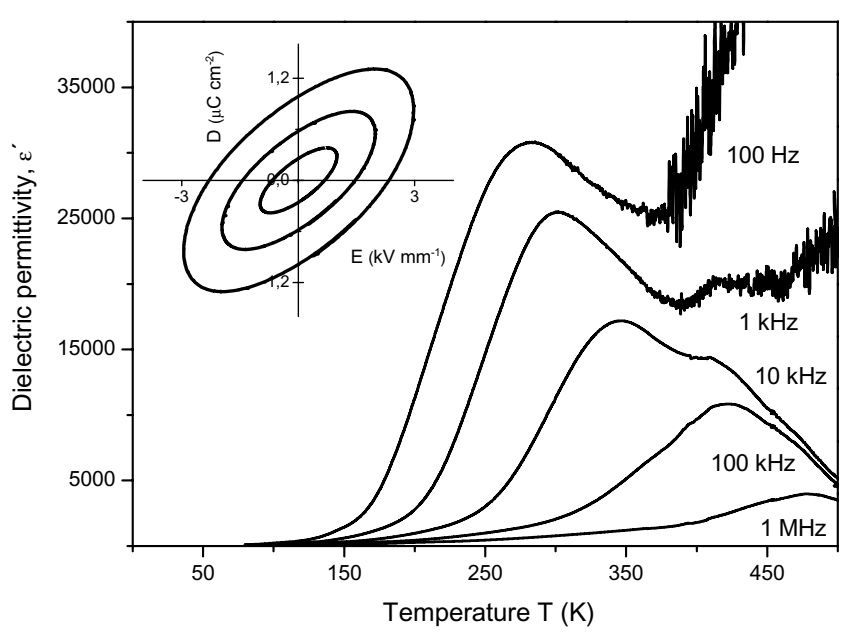

(a)

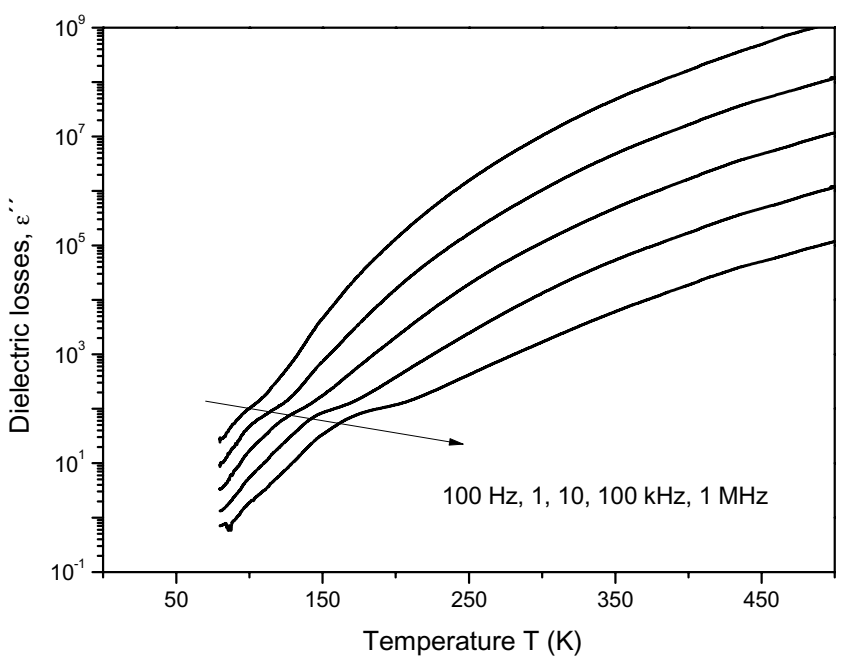

(b)

Fig. 7. Temperature dependences of the dielectric (a) permittivity, and (b) losses at several frequencies of a dense ceramic of $\mathrm{BiMnO}_{3.04}$, processed by spark plasma sintering of nanocrystalline powder obtained by mechanosynthesis. High field response at $77 \mathrm{~K}$ typical of a lossy dielectric is shown in the inset. Adapted from Ref. 39 with permission from The Royal Society of Chemistry. 
monoclinic $C 2 / c$ phases at around $475 \mathrm{~K}$. However, this is not the case.

Absence of ferroelectricity was also confirmed by measuring ferroelectric hysteresis loops at $77 \mathrm{~K}$, below the onset of conductivity. The typical behavior of lossy dielectrics with no hint of ferroelectricity was found. This is in agreement with an independent study of the lattice dynamics on samples obtained by high pressure that also showed no ferroelectricity. ${ }^{55}$

\section{The $\mathrm{BiFeO}_{3}-\mathrm{PbTiO}_{3}$ Binary System}

$\mathrm{BiFeO}_{3}$ is unambiguously multiferroic, and unlike $\mathrm{BiMnO}_{3}$, an actual example of perovskite oxides, in which independent electric and magnetic ferroic orders are obtained by placing ferroelectrically and magnetically active cations at the A-site and $\mathrm{B}$-site of the $\mathrm{ABO}_{3}$ structure, respectively. ${ }^{18}$ This compound is a proper ferroelectric with a transition temperature of $\sim 1100 \mathrm{~K}^{18,56}$ a rhombohedral structure with space group $R 3 c,{ }^{57}$ and a RT spontaneous polarization close to $100 \mu \mathrm{C}$ $\mathrm{cm}^{-2} .{ }^{58}$ Besides, the oxide is a G-type antiferromagnet with an ordering temperature of $643 \mathrm{~K} .{ }^{59}$ Crystal magnetoelectric coupling exists, and results in spin canting but also in a long range incommensurate cycloid superstructure. ${ }^{60,20}$ Weak ferromagnetism is obtained when the latter spin cycloid is destroyed by doping $\left(M_{s}=1.2 \mathrm{emu} \mathrm{g}^{-1}\right.$ for $\mathrm{Bi}_{0.7} \mathrm{Ba}_{0.3} \mathrm{FeO}_{3}$ ceramic materials). ${ }^{21}$ Therefore, it seemed an especially suitable second perovskite edge member to be combined with the $\mathrm{PbTiO}_{3}$ tetragonal perovskite, in order to obtain the targeted multiferroic MPB, not found in $\mathrm{BiMnO}_{3}-\mathrm{PbTiO}_{3}$.

Indeed the $x \mathrm{BiFeO}_{3}-(1-x) \mathrm{PbTiO}_{3}$ perovskite solid solutions series concentrates an increasing attention, and coexistence of rhombohedral $R 3 c$ and tetragonal $P 4 \mathrm{~mm}$ polymorphic phases has been described in a compositional range that varies from $0.6 \leq x \leq 0.8$ to $0.69<x<0.73$ for powders obtained by conventional solid-state synthesis. ${ }^{61-64}$ These coexisting polymorphs would be then isostructural with the edge ferroelectric oxides, which strongly suggests a ferroelectric MPB to exist. Our own results on chemically homogenous powders obtained by mechanosynthesis are shown in Fig. 8. ${ }^{65}$ A region of phase coexistence was found for $0.625 \leq x \leq 0.75$, slightly wider than that reported in Ref. 64. The reason of this discrepancy cannot be chemical homogeneity that is very high in the mechanosynthesized samples, but differences of particle size or even the temperature and/or cooling profile used in the synthesis or crystallization treatments, for these parameters have been shown to strongly affect the phase distribution in the coexistence region. $^{65}$

Note the absence of traces of second phases for all $x$ values, not only in the nanocrystalline powders that result from the mechanochemical activation, but also after the thermal treatments carried out to increase crystallinity for the structural characterization. A temperature of $1323 \mathrm{~K}$ could be used for materials with $x$ from 0 up to $x=0.75$, which had to

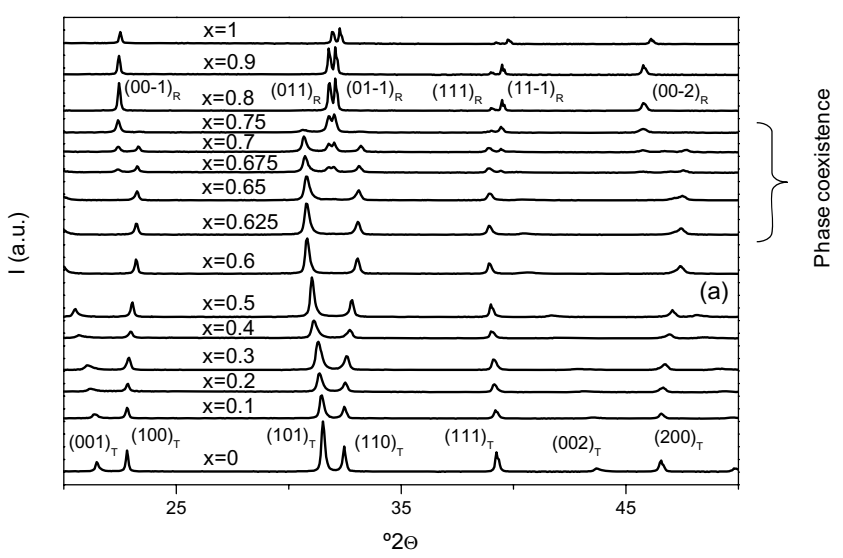

Fig. 8. XRD patterns of highly crystalline $x \mathrm{BiFeO}_{3}-(1-x) \mathrm{PbTiO}_{3}$ powders obtained by mechanosynthesis. Peaks associated with the coexisting rhombohedral $(\mathrm{R})$ and tetragonal $(\mathrm{T})$ polymorphic phases are indicated with Miller indices. Adapted from Ref. 65 with permission from The Royal Society of Chemistry.

be decreased down to 1273,1123 and $1063 \mathrm{~K}$ for $x=0.8,0.9$ and 1 , respectively to avoid triggering the perovskite decomposition.

Perovskite structure evolution across the system. Perovskite lattice parameters were obtained as a function of composition for the two polymorphs, and are shown in Fig. 9. Trends were consistent with previous reports on powders synthesized by high temperature solid-state reactions. ${ }^{63,66}$ Unlike most related binary systems, such as $\mathrm{BiScO}_{3}-\mathrm{PbTiO}_{3}$ and $\mathrm{BiMnO}_{3}-\mathrm{PbTiO}_{3}$ itself, the tetragonal distortion did not decrease on approaching the coexistence region, but continuously increased up to reach a value as high as 1.19 for $x=0.75$. This suggests a very high spontaneous polarization, as it was confirmed by electrical measurements. Perovskite cell volume is also shown in the figure. Note the large volume difference between the tetragonal and rhombohedral phases of $3.45 \AA^{3}$ within the coexistence region; to be compared with $\Delta V \sim 0.25 \AA^{3}$ for $\mathrm{BiMnO}_{3}-\mathrm{PbTiO}_{3}$, which clearly indicates a discontinuous MPB.

The actual symmetry of the rhombohedral phase close to the coexistence region is currently under debate. Evidences of an intermediate phase were firstly provided by electron diffraction, ${ }^{62}$ and subsequent Rietveld analysis of XRD data claimed this phase to be monoclinic $C c$, and to extend up to $x=0.9 .^{66}$ This has deep implications, for it opens the possibility of not only having high field phase-change responses in the system, but also enhanced low field ones by polarization rotation. $^{27,28}$

Magnetism across the system. A number of reports also addressed the evolution of magnetism across the perovskite solid solution series. Antiferromagnetic order is maintained across the whole rhombohedral and/or monoclinic fields between $x=1$ and $x=0.72$, with a Néel temperature that decreases from $630 \mathrm{~K}$ for $x=1$ down to $\sim 520 \mathrm{~K}$ for $x=0.72$, as determined from ZFC magnetization curves. ${ }^{63}$ 


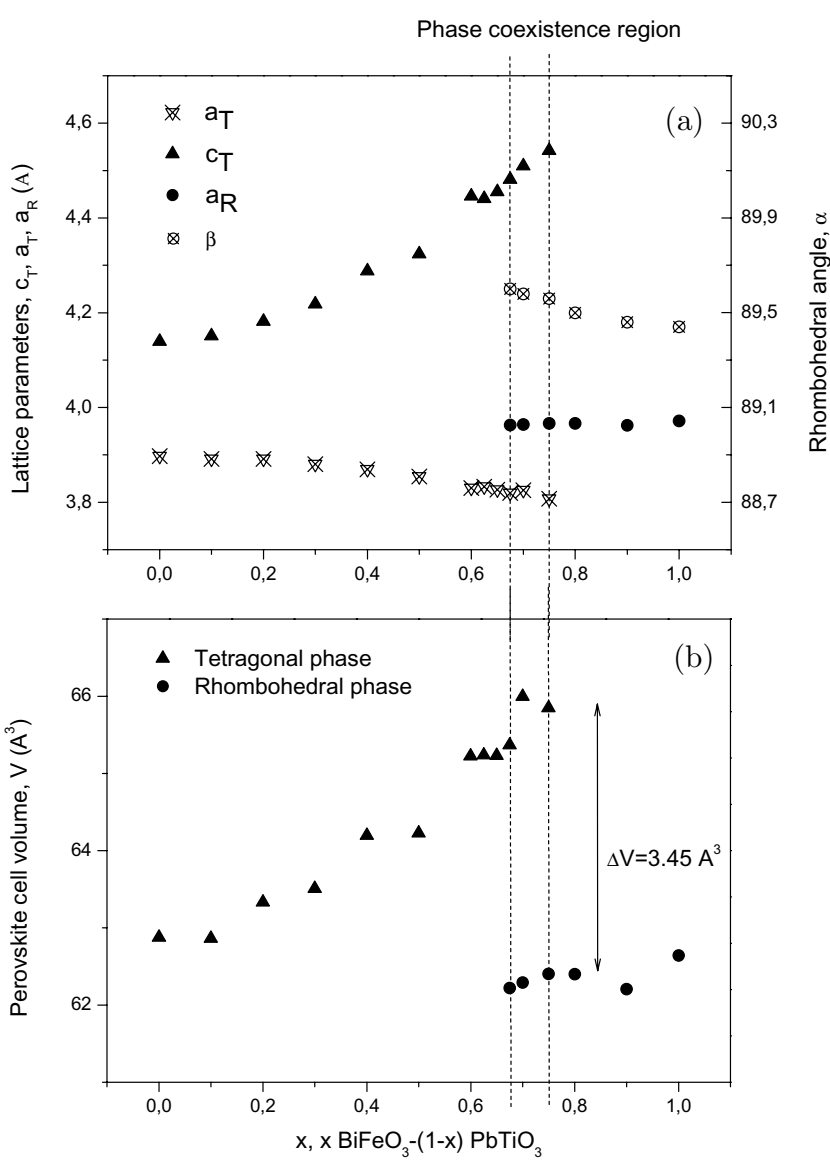

Fig. 9. (a) Perovskite lattice parameters of the tetragonal $\left(a_{T}\right.$ and $\left.c_{T}\right)$ and rhombohedral $\left(a_{R}\right.$ and $\beta$ ) polymorphic phases across the $x \mathrm{Bi}$ $\mathrm{FeO}_{3}-(1-x) \mathrm{PbTiO}_{3}$ solid solution series for the powders obtained by mechanosynthesis, and (b) evolution of the perovskite cell volume. Adapted from Ref. 65 with permission from The Royal Society of Chemistry.

G-type antiferromagnetism and a Néel temperature of $592 \mathrm{~K}$ were obtained in an independent neutron diffraction study of a material with $x=0.9{ }^{67}$ Tetragonal compositions are also antiferromagnetic at least until $x=0.45$, but with ordering temperatures below RT. ${ }^{63}$ Therefore, the coexisting polymorphs at the MPB region have well-differentiated antiferromagnetic order and Néel temperature, ${ }^{68}$ the latter being above and below RT for the rhombohedral and tetragonal phases, respectively. ${ }^{69}$

Compositions claimed to be monoclinic present a second magnetic anomaly in their ZFC curves, below the ordering temperature, ${ }^{63,70}$ which has been related to a spin reorientation transition. ${ }^{70} \mathrm{~A}$ significant irreversibility, that is divergence of the ZFC and FC curves has been recently described for a material with $x=0.675$ at the spin reorientation transition, which strongly suggests the appearance of spin canting and weak ferromagnetism at the magnetic instability. ${ }^{71}$ This effect is illustrated in Fig. 10, where the spin reorientation transition at $380 \mathrm{~K}$ is marked with an arrow. Note the persistence of significant magnetization above this temperature,

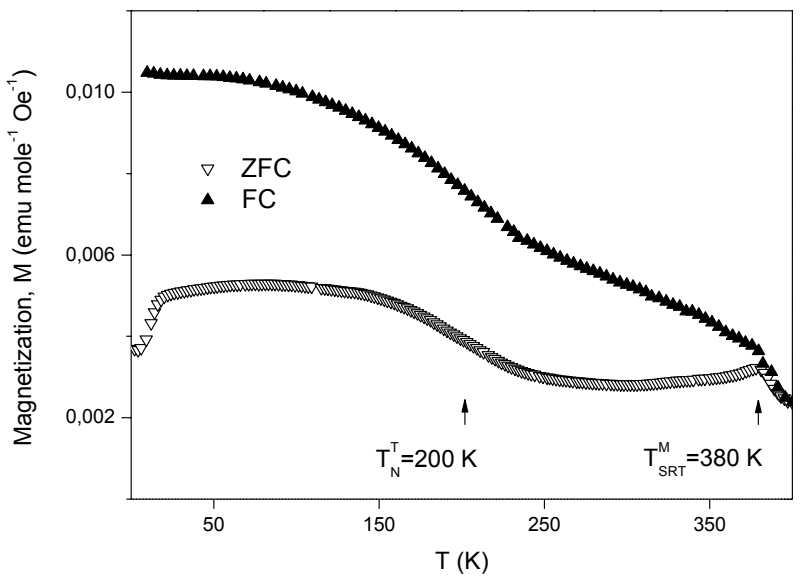

(a)

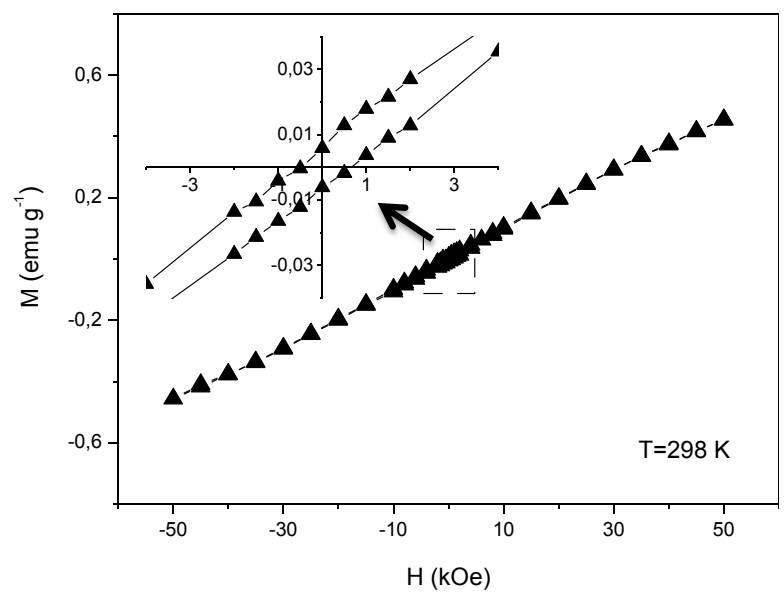

(b)

Fig. 10. Magnetic behavior of $x \mathrm{BiFeO}_{3}-(1-x) \mathrm{PbTiO}_{3}$ with $x=$ 0.675 , prepared by mechanosynthesis. (a) Magnetization between 2 and $300 \mathrm{~K}$, measured after ZFC and during subsequent FC under an applied field of $500 \mathrm{Oe}$. The spin reorientation transition of the monoclinic polymorphic phase $\left(T_{\mathrm{SRT}^{M}}\right)$, and the Néel temperature of the coexisting tetragonal phase $\left(T_{N^{T}}\right)$ are indicated. (b) RT magnetization. Reprinted with permission from Ref. 71. Copyright [2014], AIP Publishing LLC.

indicating the Néel temperature to be above the measuring range. This composition shows phase coexistence, so the magnetic anomaly associated with the ordering temperature of the tetragonal polymorph can also be seen below RT. A RT magnetization loop is also given in the figure, which shows a small, but measurable ferromagnetic component.

Ferroelectricity across the system. The ferroelectric character of the two polymorphic phases, anticipated by their crystal symmetries, was confirmed and their main parameters determined by electrical measurements, including the temperature dependence of the dielectric permittivity for determining the position of the ferroelectric transition, and ferroelectric hysteresis loops in ceramics samples processed from the nanocrystalline powders. ${ }^{71,72}$ 
Nevertheless, the ferroelectric transition temperatures were also determined by differential thermal analysis in the powdered samples obtained by mechanosynthesis. ${ }^{65}$ Distinctive endothermic reversible effects that could be directly related to the transition were found for all compositions from $x=0$ to 1 . A tricritical point then does not seem to exist in the binary system, and the ferroelectric transition is first order all across the solid solution series. The evolution of $T_{C}$ with $x$ is shown in Fig. 11, where a continuous increase with $x$, along with a distinctive change of the slope within the phase coexistence region is evident.

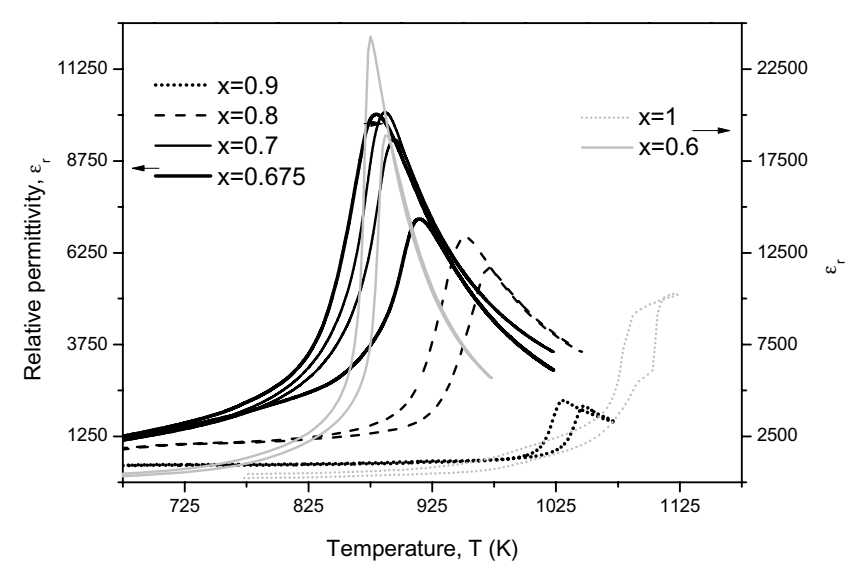

(a)

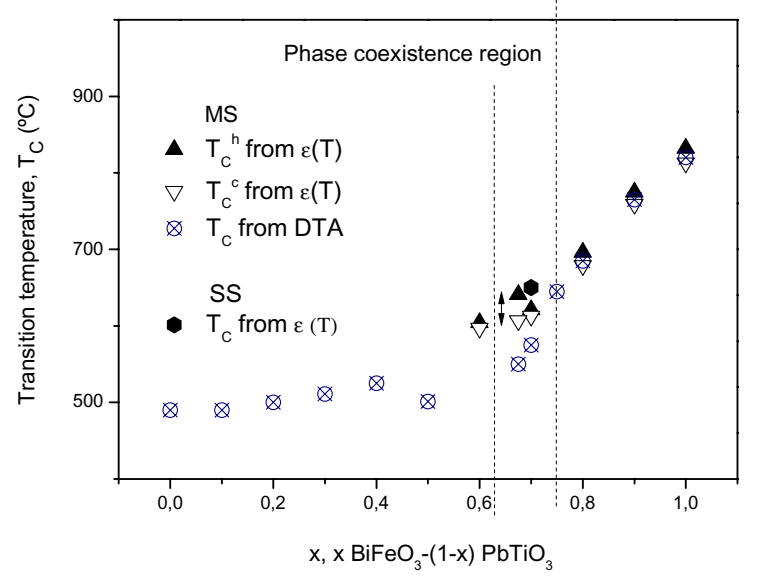

(b)

Fig. 11. (a) Temperature dependence of the relative permittivity at $1 \mathrm{MHz}$ of six $x \mathrm{BiFeO}_{3}-(1-x) \mathrm{PbTiO}_{3}$ ceramics with $x=1,0.9,0.8$, $0.7,0.675$ and 0.6 , processed from the nanocrystalline powders obtained by mechanosynthesis (adapted with permission from Ref. 71. Copyright [2014], AIP Publishing LLC), and (b) ferroelectric transition temperatures $\left(T_{C^{h}}\right.$ determined on heating, $T_{C^{c}}$ on cooling, and $T_{C}$ not specified) across the $x \mathrm{BiFeO}_{3}-(1-x) \mathrm{PbTiO}_{3}$ solid solution series for samples obtained by mechanosynthesis: MS (from Refs. 65 and 71), and by conventional means: SS (from Ref. 73), experimentally determined either by differential thermal analysis: DTA, or from the temperature dependence of permittivity: $\varepsilon(T)$.
The ferroelectric transition was further studied by measuring the temperature dependence of the dielectric permittivity, after the processing of ceramics by hot pressing of the nanocrystalline powders for $1 \geq x \geq 0.65$, or by spark plasma sintering of the same powder followed by a thermal treatment for $x<0.65$. The latter procedure was necessary for compositions in the tetragonal field, next to the MPB because of the very high tetragonal distortion that made hot pressed samples to disintegrate during cooling. Like for $\mathrm{BiMnO}_{3}-$ $\mathrm{PbTiO}_{3}$, conductivity was an issue, this time associated with the presence of oxygen vacancies and $\mathrm{Fe}^{2+}$, so as both low temperature electron hopping between $\mathrm{Fe}^{2+}$ and $\mathrm{Fe}^{3+}$ and high temperature ionic conduction, along with a number of related dielectric relaxations contributed to the electrical response. As a consequence, a stringent control of the processing conditions was necessary to obtain materials with minimized conduction related phenomena (and also enhanced dielectric breakdown resistance), which allowed the ferroelectric parameters to be determined. Details of this sample tailoring can be found in Ref. 71 .

The temperature dependence of the permittivity for six materials covering the rhombohedral/monoclinic field, the coexistence region, and a tetragonal composition with very high tetragonal distortion are given in Fig. 11 at $1 \mathrm{MHz}$. The obtained evolution of $T_{C}$ with $x$ is compared with that of powders measured by DTA in the same figure. The transition temperature for a ceramic material with $x=0.7$, processed from powders synthesized by conventional solid-state reactions, and taken from an independent report is included for completeness. ${ }^{73}$ Note the discrepancy between the transition temperatures of powders and ceramics in the coexistence region that suggests a higher percentage of tetragonal phase in the former samples for a given composition, in agreement with XRD results. ${ }^{65,72}$ This is most probably an effect of the ceramic stress field, and nicely illustrates how close the two polymorphs are in the free energy space.

Ferroelectric hysteresis loops for the same six materials are shown in Fig. 12. As processed ceramics did not show ferroelectric switching up to breakdown fields, and a thermal treatment consisting of heating above the Curie temperature, followed by quenching was required to obtain saturated loops. This has been proposed to result in the release of the ferroelectric domain walls, initially clamped by the presence of aligned $\mathrm{Fe}^{2+}$-oxygen vacancy dipolar defects. ${ }^{71,72}$ Indeed, this treatment was not efficient enough to obtain fully saturated loops for $x=1$, that is $\mathrm{BiFeO}_{3}$, because the transition temperature of $1100 \mathrm{~K}$ could not be exceeded in this case. Perovskite decomposition was already triggered at a temperature as low as $1063 \mathrm{~K}$ for the multiferroic edge member. A somehow similar problem was found for $x=0.6$, whose very high transition strain characteristic of the tetragonal phase made the quenching treatment to result in microcracking. However, saturated loops were obtained all across the rhombohedral/monoclinic field, and within the coexistence region. 


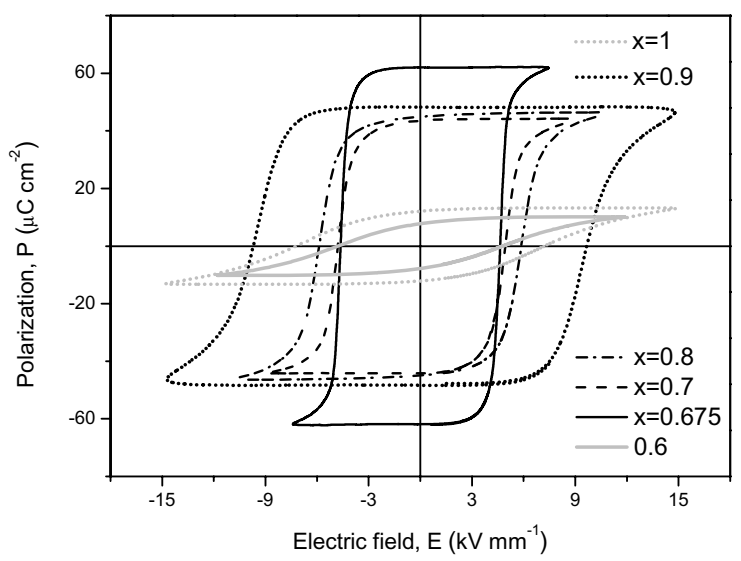

(a)

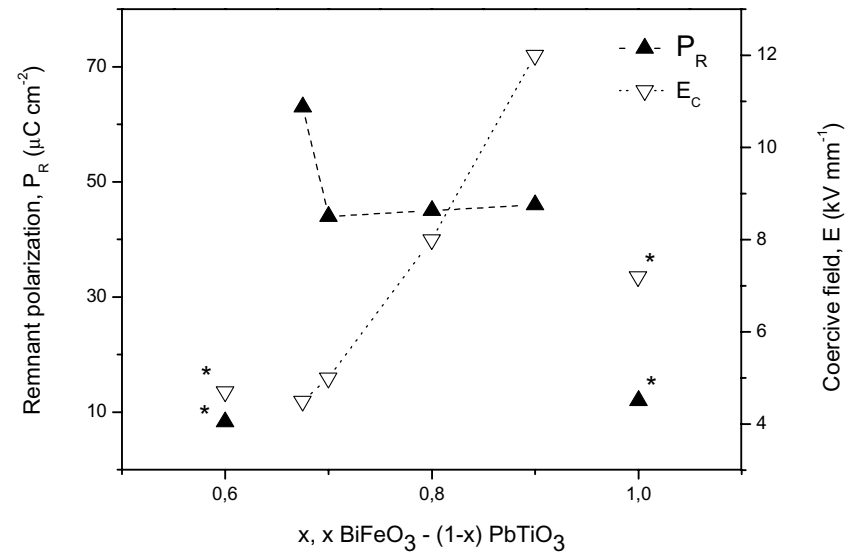

(b)

Fig. 12. (a) Ferroelectric hysteresis loops at $0.1 \mathrm{~Hz}$ of six $x \mathrm{BiFeO}_{3}-(1-x) \mathrm{PbTiO}_{3}$ ceramics with $x=1,0.9,0.8,0.7,0.675$ and 0.6 , processed from the nanocrystalline powders obtained by mechanosynthesis (from Ref. 71 but for $x=0.7$ that is unpublished), and (b) the obtained ferroelectric parameters $\left(P_{R}\right.$ remnant polarization, and $E_{C}$ coercive field) across the $x \mathrm{BiFeO}_{3}-(1-x) \mathrm{PbTiO}_{3}$ solid solution series. $*$ From unsaturated loops.

The ferroelectric parameters across the binary system are given in the same figure. Recall that those of $x=1$ and $x=$ 0.6 correspond to unsaturated loops. Remnant polarization, $P_{R}$, seemed to maintain a constant value around $45 \mu \mathrm{C} \mathrm{cm}{ }^{-2}$ for materials with $x=0.9,0.8$ and 0.7 and rhombohedral or monoclinic symmetry. However, the coercive field continuously decreased from $12 \mathrm{kV} \mathrm{mm}^{-1}$ for $x=0.9$, down to 8 and $5 \mathrm{kV} \mathrm{mm}^{-1}$ for $x=0.8$ and 0.7 , respectively. A fairly constant spontaneous polarization thus exists in this compositional range, which would be $\sim 90 \mu \mathrm{C} \mathrm{cm}^{-2}$ under the assumption that only inversion domains contribute to switching (we will see later this to be the case). The figure is very close to that reported for rhombohedral $\mathrm{BiFeO}_{3}$ single crystals. ${ }^{58}$ However, coercive field does change, which suggests a strong modification of the domain configuration with increasing $x$. This might be a consequence of the reported rhombohedral to monoclinic transition for $x=0.9^{66}$ that could be also responsible of the significant modification of the dielectric anomaly when $x$ was decreased from 0.9 down to 0.8 (see Fig. 11). It is worth noting that the ferroelectric transition is from an orthorhombic Pnma phase to a rhombohedral $R 3 c$ one for $x=1$, but from a cubic $P m 3 m$ phase to monoclinic $C c$ for $x=0.73$. $^{66}$

A main result of the electrical study was the significant increase of $P_{R}$ when entering the phase coexistence region, up to a value as high as $63 \mu \mathrm{C} \mathrm{cm}{ }^{-2}$, while the coercive field remained basically unchanged. This cannot be associated with a sudden increase of the spontaneous polarization in the monoclinic component, for the previous evolution with $x$ does not indicate it. It cannot be associated with the tetragonal component either, for only incipient switching is obtained in this phase at the applied fields, as shown by the loop of the material with $x=0.6$. A possibility might be the onset of a ferroelectric/ferroelastic contribution, but this should be accompanied by an increase of $E_{C}$. Besides, the RT electromechanical response of ceramics with $x=0.675$, shown for fields up to $2 \mathrm{kV} \mathrm{mm}^{-1}$ in Fig. 13, is very linear and hysteresis-free, which seems to exclude this mechanism. Therefore, this very high number can only be understood if a significant phase-change contribution to switching is assumed. ${ }^{71,72}$ Indeed, a distinctive phase-change electromechanical response was revealed when temperature was raised above $425 \mathrm{~K}$, and the threshold field was decreased below the applied ones. ${ }^{72}$

The evolution of the electrical and electromechanical linear coefficients across the perovskite solid solution series was outstanding too. ${ }^{71}$ Permittivity and the charge piezoelectric coefficient are also given in Fig. 13. A distinctive enhancement of the $\varepsilon_{33}$ dielectric permittivity and the $d_{33}$ piezoelectric coefficient at the phase coexistence region, similar to that described at the ferroelectric instabilities of $\mathrm{Pb}(\mathrm{Zr}, \mathrm{Ti}) \mathrm{O}_{3}$ and a range of related perovskite solid solutions, was found. ${ }^{27,74}$ In the case of $\mathrm{Pb}(\mathrm{Zr}, \mathrm{Ti}) \mathrm{O}_{3}$, this enhancement is known to be the combination of two effects: (1) the presence of a lattice transverse instability at the monoclinic $\mathrm{Cm}-$ tetragonal $P 4 \mathrm{~mm}$ boundary, ${ }^{28}$ and (2) an enhanced ferroelectric/ferroelastic domain wall activity within the monoclinic phase. ${ }^{75}$ In the case of $\mathrm{BiFeO}_{3}-\mathrm{PbTiO}_{3}$, however, no ferroelectric/ferroelastic contribution to the electromechanical response has been found up to electric fields as high as $2 \mathrm{kV}$ $\mathrm{mm}^{-1}$. Therefore, the enhancement of the piezoelectric coefficient at the MPB must only reflect the presence of lattice transverse softening and thus, the enabling of the associated polarization rotation mechanism in the multiferroic system. Indeed, the actual figure of $87 \mathrm{pC} \mathrm{N}^{-1}$ for $\mathrm{MPB} \mathrm{BiFeO}_{3}-$ $\mathrm{PbTiO}_{3}$ favorably compares with the single crystal contribution to the coefficient of $\mathrm{Pb}(\mathrm{Zr}, \mathrm{Ti}) \mathrm{O}_{3}$ ceramics, reported to be $80 \mathrm{pC} \mathrm{N}^{-1}$ (raised up to $245 \mathrm{pC} \mathrm{N}^{-1}$ by domain wall contribution). ${ }^{76}$ Note that this mechanism requires the presence of the monoclinic polymorph, so these results support 


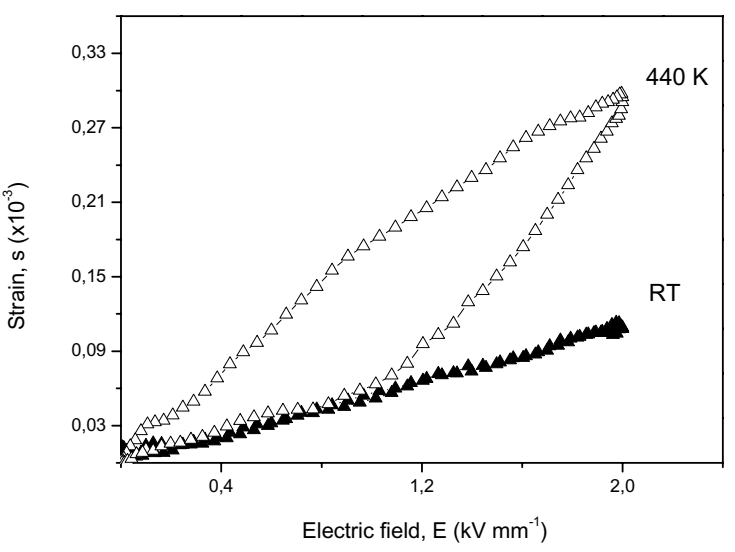

(a)

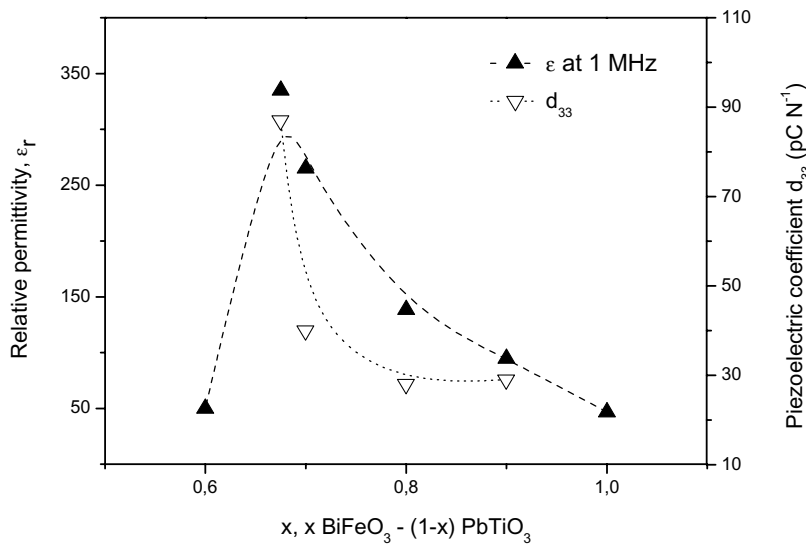

(b)

Fig. 13. (a) Electric field induced strain for one $x \mathrm{BiFeO}_{3}-(1-x) \mathrm{PbTiO}_{3}$ ceramic with $x=0.675$, processed by hot pressing of nanocrystalline powders obtained by mechanosynthesis (adapted with permission from Ref. 72. Copyright [2012], AIP Publishing LLC), and (b) relative permittivity $\left(\varepsilon_{r}\right)$ and charge piezoelectric coefficient $\left(d_{33}\right)$ across the $x \mathrm{BiFeO}_{3}-(1-x) \mathrm{PbTiO}_{3}$ solid solution series (adapted with permission from Ref. 71. Copyright [2014], AIP Publishing LLC).

the structural characterizations that indicated the presence of the $C c$ phase.

A similar exercise can be done with permittivity. The figure for $\mathrm{MPB} \mathrm{BiFeO}_{3}-\mathrm{PbTiO}_{3}$ of 335 is also comparable to the single crystal contribution to the permittivity of $\mathrm{Pb}(\mathrm{Zr}, \mathrm{Ti})-\mathrm{O}_{3}$ ceramics, this reported to be 350 (raised up to 1200 by domain wall contributions). ${ }^{76}$ It is clear that inversion domains do move in $\mathrm{BiFeO}_{3}-\mathrm{PbTiO}_{3}$; there would be no ferroelectric switching otherwise, but the very high coercive fields, one order of magnitude larger to those of $\mathrm{Pb}(\mathrm{Zr}, \mathrm{Ti}) \mathrm{O}_{3}$, hint their very low contribution to the linear coefficient.

Analysis of multiferroism and the potential magnetoelectric response. In summary, and unlike $\mathrm{BiMnO}_{3}-\mathrm{PbTiO}_{3}$, the perovskite $x \mathrm{BiFeO}_{3}-(1-x) \mathrm{PbTiO}_{3}$ solid solution series shows multiferroic states for all compositions from $x=1$ down to 0.45 . This comprises the phase coexistence region between the monoclinic and tetragonal polymorphs that is thus a multiferroic phase boundary like targeted. A distinctive characteristic of the system is that both polymorphic phases have the electrical ordering temperatures above RT, but only the monoclinic one has the magnetic ordering temperature above RT, while the Néel temperature of the tetragonal component is below $298 \mathrm{~K}$.

Besides, strong RT phase-change electrical and electromechanical responses have been found for $\mathrm{BiFeO}_{3}-\mathrm{PbTiO}_{3}$ ceramics at the MPB. This involves the partially reversible electrical field induced transformation between a canted antiferromagnet and a paramagnet and thus, a distinctive phasechange magnetoelectric response can be expected.

Moreover, the system shows transverse lattice softening at the multiferroic monoclinic-tetragonal boundary, and related enhancement of permittivity and piezoelectric coefficient by the polarization rotation mechanism. An analogous enhancement of the magnetoelectric coefficients might be expected if the ferroelectric and magnetic orderings are coupled like in $\mathrm{BiFeO}_{3} .{ }^{19,20}$ This coupling has been suggested to exist in $x \mathrm{BiFeO}_{3}-(1-x) \mathrm{PbTiO}_{3}$ with $x=0.73$, based on the observation of a dielectric anomaly at the antiferromagnetic transition. ${ }^{77}$

\section{Final Considerations and Future Trends}

Here we have put together our investigations on two systems, whose phase diagrams were, in principle, liable to show multiferroic MPBs. Design concept was quite straightforward, namely to form perovskite solid solutions with one edge member of crystal symmetry other than tetragonal, which included stereochemically active $\mathrm{Bi}^{3+}$ at the A-site and a magnetically active cation in the B-site, and tetragonal $\mathrm{PbTiO}_{3}$ as the second edge member. $\mathrm{Pb}$-based perovskites like $\mathrm{Pb}\left(\mathrm{Fe}_{1 / 2} \mathrm{Nb}_{1 / 2}\right) \mathrm{O}_{3}$ were avoided, for the low concentration of the magnetic species anticipated very low magnetic ordering temperatures.

Indeed, MPBs were found for both cases, but of very different nature. A multiferroic instability does not exist for $\mathrm{BiMnO}_{3}-\mathrm{PbTiO}_{3}$, but a MPB between a ferroelectric tetragonal $P 4 \mathrm{~mm}$ polymorphic phase and a nonpolar orthorhombic Pnma phase. Magnetic order sets in for the latter polymorph but at very low temperature. Besides, tetragonal phases next to the MPB transform into the orthorhombic phase on cooling. Therefore, multiferroic states do not exist across the system at any temperature.

Contrarily, a multiferroic instability unambiguously exists for $\mathrm{BiFeO}_{3}-\mathrm{PbTiO}_{3}$ that shows a MPB between ferroelectric, canted antiferromagnets of monoclinic $C c$ and tetragonal $\mathrm{P} 4 \mathrm{~mm}$ symmetries. Furthermore, electric field induced phase transformations between the two polymorphic phases, as well as lattice transverse softening at the monoclinic-tetragonal boundary have been demonstrated. A main issue of this 
multiferroic instability is its antiferromagnetic nature, and the very weak ferromagnetism provided by the spin canting. Novel systems thus need to be found with a ferromagnetic character, or at least enhanced weak ferromagnetism.

A promising candidate that we are currently investigating is the $\mathrm{BiFeO}_{3}-\mathrm{BiMnO}_{3}-\mathrm{PbTiO}_{3}$ ternary system, and specifically the line across the phase diagram that joins the morphotropic phase boundaries of the $x \mathrm{BiFeO}_{3}-(1-x) \mathrm{PbTiO}_{3}$ and $x \mathrm{BiMnO}_{3}-(1-x) \mathrm{PbTiO}_{3}$ perovskite solid solution series, located at $x=0.7$ and $x=0.4$, respectively. It has been proposed after an study of the strongly related ternary $\mathrm{BiScO}_{3}-\mathrm{BiFeO}_{3}-\mathrm{PbTiO}_{3}$ that a Vegard-type relationship exists for the MPB compositions, so as the line that joins the MPBs of two binary $\mathrm{BiMO}_{3}-\mathrm{PbTiO}_{3}$ and $\mathrm{BiM}^{\prime} \mathrm{O}_{3}-\mathrm{PbTiO}_{3}$ systems across the phase diagram of the corresponding $\mathrm{BiMO}_{3}-\mathrm{BiM}^{\prime} \mathrm{O}_{3}-\mathrm{PbTiO}_{3}$ ternary is a line of MPBs. ${ }^{40}$ Indeed,

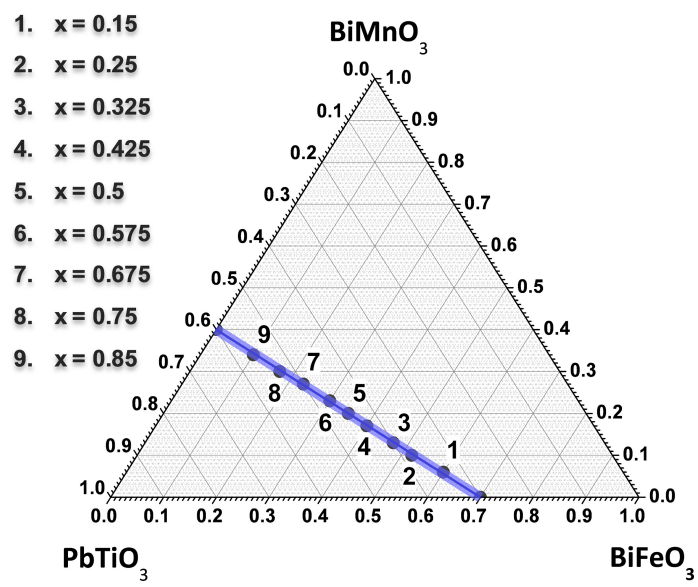

(a)
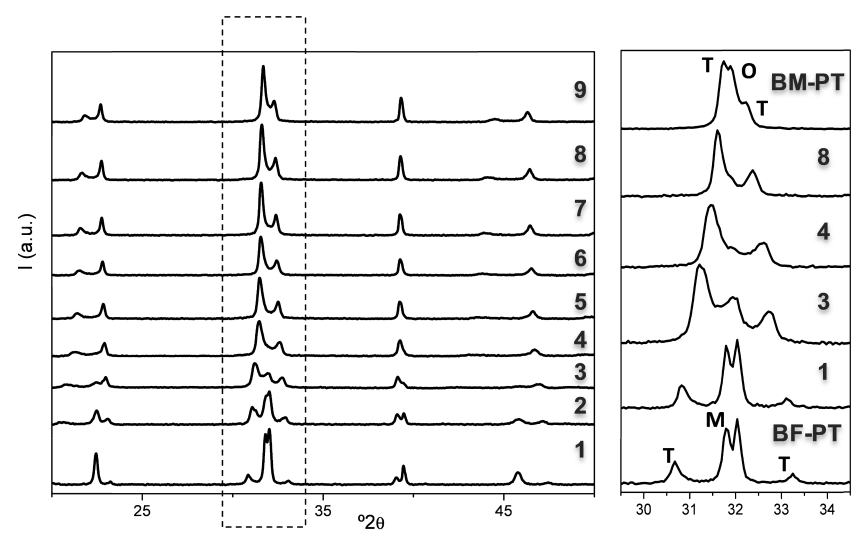

${ }^{\circ} 2 \Theta$

(b)

Fig. 14. (a) Line of MPBs across the $\mathrm{BiFeO}_{3}-\mathrm{BiMnO}_{3}-\mathrm{PbTiO}_{3}$, expressed as $x\left(0.4 \mathrm{BiMnO}_{3}-0.6 \mathrm{PbTiO}_{3}\right)-(1-x)\left(0.7 \mathrm{BiFeO}_{3}-0.3 \mathrm{PbTiO}_{3}\right)$. (b) XRD patterns of selected highly crystalline powders along this line. Peaks associated with the coexisting monoclinic, tetragonal and orthorhombic polymorphic phases are indicated with $\mathrm{M}, \mathrm{T}$ and O, respectively. Adapted from Ref. 78 with permission from The Royal Society of Chemistry. this is the case for $\mathrm{BiFeO}_{3}-\mathrm{BiMnO}_{3}-\mathrm{PbTiO}_{3}$, for which phase coexistence, initially between a rhombohedral $R 3 c$ and tetragonal $P 4 \mathrm{~mm}$ polymorphic phases that subsequently evolves towards a coexistence between tetragonal $P 4 \mathrm{~mm}$ and orthorhombic Pnma ones, has been recently described along such a line. ${ }^{78}$ This is illustrated in Fig. 14.

A consistent evolution of the magnetism and ferroelectricity has also been found along the line, with distinctive features at selected compositions, such as decrease of the temperature of the spin reorientation transition, accompanied by an increase of the irreversibility and thus, an enhancement of the weak magnetism. ${ }^{78}$ Moreover, an unambiguous RT phase-change magnetoelectric response has been obtained for a chemically engineered composition at a monoclinic $C c$ tetragonal $P 4 \mathrm{~mm}$ boundary, which also shows a significant magnetoelectric linear coefficient by polarization rotation. ${ }^{79}$

\section{Acknowledgments}

This work has been funded by Spanish MINECO through the MAT2014-58816-R and MAT2011-2370 projects. C. M.F-P. also acknowledges the financial aid of the Spanish FPI Program (BES 2012-053017). Technical support all along the research by Ms. I. Martínez and Ms. M. M. Antón at ICMMCSIC is recognized.

\section{References}

${ }^{1}$ W. Eerenstein, N. Mathur and J. F. Scott, Multiferroic and magnetoelectric materials, Nature 442, 759 (2006).

${ }^{2}$ G. Lawes and G. Srinivasan, Introduction to magnetoelectric coupling and multiferroic films, J. Phys. D: Appl. Phys. 44(24), 243001 (2011).

${ }^{3}$ J. F. Scott, Room temperature multiferroic magnetoelectrics, $N P G$ Asia Mater. 5, E72 (2013).

${ }^{4} \mathrm{M}$. Bibes and A. Barthelemy, Multiferroics: Towards a magnetoelectric memory, Nature Mater. 7(6), 425 (2008).

5 J. F. Scott, Applications of magnetoelectrics, J. Mater. Chem. 22 (11), 4567 (2012).

${ }^{6} \mathrm{D}$. Sando, A. Barthelemy and M. Bibes, $\mathrm{BiFeO}_{3}$ epitaxial thin films and devices: Past, present and future, J. Phys.: Condens. Matter. 26(47), 473201 (2014).

${ }^{7}$ E. Ascher, H. Rieder, H. Schmid and H. Stössel, Some properties of ferromagnetoelectric nickel-iodine boracite $\mathrm{Ni}_{3} \mathrm{~B}_{7} \mathrm{O}_{13} \mathrm{I}$, J. Appl. Phys. 37, 1404 (1966).

${ }^{8}$ T. Kimura, T. Goto, H. Shintani, K. Ishizaka, T. Arima and Y. Tokura, Magnetic control of ferroelectric polarization, Nature $\mathbf{4 2 6}$, 55 (2003).

${ }^{9}$ N. Hur, S. Park, P. A. Sharma, J. S. Ahn, S. Guha and S. W. Cheong, Electric polarization reversal and memory in a multiferroic material induced by magnetic fields, Nature 429, 392 (2004).

${ }^{10} \mathrm{~S}$. W. Cheong and M. Mostovoy, Multiferroics: A magnetic twist for ferroelectricity, Nat. Mater. 6(1), 13 (2007).

${ }^{11}$ T. Kimura, Y. Sekio, H. Nakamura, T. Siegrist and A. P. Ramirez, Cupric oxide as an induced-multiferroic with high- $\mathrm{T}_{C}$, Nat. Mater. 7, 291 (2008). 
${ }^{12}$ Y. Kitagawa, Y. Hiraoka, T. Honda, T. Ishikura, H. Nakamura and T. Kimura, Low-field magnetoelectric effect at room temperature, Nat. Mater. 9, 797 (2010).

${ }^{13}$ K. R. S. Preethi-Meher, C. Martin, V. Caignaert, F. Damay and A. Maignan, Multiferroics and magnetoelectrics: A comparison between some chromites and cobaltites, Chem. Mater. 26, 830 (2014).

${ }^{14} \mathrm{M}$. Fiebig, Revival of the magnetelectric effect, J. Phys. D: Appl. Phys. 38(8), R123 (2005).

${ }^{15}$ R. E. Cohen, Origin of ferroelectricity in perovskite oixdes, Nature 358(6382), 136 (1992).

${ }^{16} \mathrm{~N}$. A. Hill, Why are there so few magnetic ferroelectrics? J. Phys. Chem. B 104(29), 6694 (2000).

${ }^{17}$ J. Wang, J. B. Neaton, H. Zheng, V. Nagarajan, S. B. Ogale, B. Liu, D. Viehland, V. Vaithyanathan, D. G. Schlom, U. V. Waghmare, N. A. Spaldin, K. M. Rabe, M. Wuttig and R. Ramesh, Epitaxial $\mathrm{BiFeO}_{3}$ multiferroic thin film heterostructures, Science 299(5613), 1719 (2003).

${ }^{18}$ G. Catalan and J. F. Scott, Physics and Applications of bismuth ferrite, Adv. Mater. 21(24), 2463 (2009).

${ }^{19}$ T. Zhao, A. Scholl, F. Zavaliche, K. Lee, M. Barry, A. Doran, M. P. Cruz, Y. H. Chu, C. Ederer, N. A. Spaldin, R. R. Das, D. M. Kim, S. H. Baek, C. B. Eom and R. Ramesh, Electrical control of antiferromagnetic domains in multiferroic $\mathrm{BiFeO}_{3}$ films at room temperature, Nat. Mater. 5, 823 (2006).

${ }^{20}$ D. Lebeugle, D. Colson, A. Forget, M. Viret and A. M. Bataille, Electric-field-induced spin flop in $\mathrm{BiFeO}_{3}$ single crystals at room temperature, Phys. Rev. Lett. 100, 227602 (2008).

${ }^{21}$ V. A. Khomchenko, D. A. Kiselev, J. M. Vieira, L. Jian, A. L. Kholkin, A. M. L. Lopes, Y. G. Pogorelov, J. P. Araujo and $\mathrm{M}$. Maglione, Effect of diamagnetic $\mathrm{Ca}, \mathrm{Sr}, \mathrm{Pb}$ and $\mathrm{Ba}$ substitution on the crystal structure and multiferroic properties of the $\mathrm{BiFeO}_{3}$ perovskite, J. Appl. Phys. 103, 024105 (2008).

${ }^{22}$ S. M. Wu, S. A. Cybart, P. Yu, M. D. Rossell, J. X. Zhang, R. Ramseh and R. C. Dynes, Reversible electric control of exchange bias in a multiferroic field-effect device, Nat. Mater. 9, 756 (2010).

${ }^{23}$ D. M. Evans, A. Schilling, A. Kumar, D. Sanchez, N. Ortega, M. Arredondo, R. S. Katiyar, J. M. Gregg and J. F. Scott, Magnetic switching of ferroelectric domains at room temperature in multiferroic PZTFT, Nat. Commun. 4, 1534 (2013).

${ }^{24}$ D. Sánchez, N. Ortega, A. Kumar, G. Sreenivasulu, R. S. Katiyar, J. F. Scott, D. M. Evans, M. Arredondo-Arechavala, A. Schilling and J. M. Gregg, Room-temperature single phase multiferroic magnetoelectrics: $\mathrm{Pb}(\mathrm{Fe}, \mathrm{M})_{x}(\mathrm{Zr}, \mathrm{Ti})_{1-x} \mathrm{O}_{3}[\mathrm{M}=\mathrm{Ta}, \mathrm{Nb}]$, J. Appl. Phys. 113, 074105 (2013).

${ }^{25} \mathrm{O}$. Dieguez and J. Iniquez. First-principles investigation of morphotropic transitions and phase-change functional responses in $\mathrm{BiFeO}_{3}-\mathrm{BiCoO}_{3}$ multiferroic solid solutions, Phys. Rev. Lett. 107, 057601 (2011).

${ }^{26}$ K. Oka, T. Koyama, T. Ozaaki, S. Mori, Y. Shimakawa and K. Azuma, Polarization rotation in the monoclinic perovskite $\mathrm{BiCo}_{1-x} \mathrm{Fe}_{x} \mathrm{O}_{3}$, Angew. Chem. Int. Ed. 51, 7977 (2012).

${ }^{27}$ R. Guo, L. E. Cross, S. E. Park, B. Noheda, D. E. Cox and G. Shirane, Origin of the high piezoelectric response in $\mathrm{PbZr}_{1-x} \mathrm{Ti}_{x} \mathrm{O}_{3}$, Phys. Rev. Lett. 84, 5423 (2000).

${ }^{28}$ D. Damjanovic, Comments on origins of enhanced piezoelectric properties in ferroelectrics, IEEE Trans. Ultrason. Ferroelectr. Freq. Control 56, 1574 (2009).

${ }^{29}$ M. Algueró, J. Ricote, T. Hungría and A. Castro, High-sensitivity piezoelectric, low-tolerance-factor perovskites by mechanosynthesis, Chem. Mater. 19(20), 4982 (2007).
${ }^{30}$ A. Moreira dos Santos, A. K. Cheetham, T. Atou, Y. Syono, Y. Yamaguchi, K. Ohoyama and H. Chiba, Orbital ordering as the determinant for ferromagnetism in biferroic $\mathrm{BiMnO}_{3}$, Phys. Rev. $B$ 66, 064425 (2002).

${ }^{31}$ T. Atou, H. Chiba, k. Ohoyama, Y. Yamaguchi and Y. Syono, Structure determination of ferromagnetic perovskite $\mathrm{BiMnO}_{3}, J$. Solid State Chem. 145, 639 (1999).

${ }^{32}$ A. Moreira dos Santos, S. Parashar, A. R. Raju, Y. S. Zhao, A. K. Cheetham and C. N. R. Rao, Evidence for the likely occurrence of magnetoferroelectricity in the simple perovskite $\mathrm{BiMnO}_{3}$, Solid State Commun. 122, 49 (2002).

${ }^{33}$ A. Sharan, J. Lettieri, Y. Jia, W. Tian, X. Pan, X. Pan, D.G. Scholm and V. Gopalan, Bismuth manganite: A multiferroic with a large nonlinear optical response, Phys. Rev. B 69, 214109 (2004).

${ }^{34}$ R. Seshadri and N. A. Hill, Visualizing the role of Bi 6s "lone pairs" in the off-center distortion in ferromagnetic $\mathrm{BiMnO}_{3}$, Chem. Mater. 13, 2892 (2001).

${ }^{35}$ D. I. Woodward and I. M. Reaney, A structural study of ceramics in the $\left(\mathrm{BiMnO}_{3}\right)_{x}-\left(\mathrm{PbTiO}_{3}\right)_{1-x}$ solid solution series, J. Phys.: Condens. Matter 16, 8823 (2004).

${ }^{36}$ A. A. Bokov and Z. G. Ye, Dielectric relaxation in relaxor ferroelectrics, J. Adv. Dielect. 2(2), 1241010 (2012).

${ }^{37}$ A. Castro, E. Vila, R. Jiménez, J. Hernández-Velasco, T. Hungría and M. Algueró, Synthesis, structural characterization and properties of perovskites belonging to the $\mathrm{x} \mathrm{BiMnO}_{3}-(1-\mathrm{x}) \mathrm{PbTiO}_{3}$ system, Chem. Mater. 22, 541 (2010).

${ }^{38}$ T. Hungría, C. Correas, F. Houdellier, O. Peña, E. Vila and A. Castro, Study of nanocrystalline $\mathrm{BiMnO}_{3}-\mathrm{PbTiO}_{3}$ : Synthesis, structural elucidation, and magnetic characterization of the whole solid solution, Chem. Eur. J. 18, 9075 (2012).

${ }^{39}$ A. Castro, C. Correas, O. Peña, A. R. Landa-Cánovas, M. Algueró, H. Amorín, M. Dollé, E. Vila and T. Hungría, Nanostructured $\mathrm{BiMnO}_{3+\delta}$ obtained at ambient pressure: Analysis of its multiferroicity, J. Mater. Chem. 22, 9928 (2012).

${ }^{40}$ M. Algueró, P. Ramos, R. Jiménez, H. Amorín, E. Vila and A. Castro, High temperature piezoelectric $\mathrm{BiScO}_{3}-\mathrm{PbTiO}_{3}$ synthesized by mechanochemical methods, Acta Mater. 60(3), 1174 (2012).

${ }^{41}$ T. Kimura, S. Kawamoto, I. Yamada, M. Azuma, M. Takano and Y. Tokura, Magnetocapacitance effect in multiferroic $\mathrm{BiMnO}_{3}$, Phys. Rev. B 67, 180401 (2003).

${ }^{42}$ E. Montanari, G. Calestani, A. Migliori, M. Dapiaggi, F. Bolzoni, R. Cabassi and E. Gilioli, High-temperature polymorphism in metastable $\mathrm{BiMnO}_{3}$, Chem. Mater. 17, 6457 (2005).

${ }^{43}$ A. A. Belik, H. Yusa, N. Hirao, Y. Ohishi and F. TakayamaMuromachi, Peculiar high pressure behavior of $\mathrm{BiMnO}_{3}$, Inorg. Chem. 48, 1000 (2009).

${ }^{44}$ A. A. Belik, K. Kodama, N. Igawa, S. Shamoto, K. Kosuda and E. Takayama-Muromachi, Crystal and magnetic structures and properties of $\mathrm{BiMnO}_{3 \pm \delta}$, J. Am. Chem. Soc. 132, 8137 (2010).

${ }^{45}$ M. Ahart, M. Somayazulu, R. E. Cohen, P. Ganesh, P. Dera, H. K. Mao, R. J. Hemley, Y. Ren, P. Liermann and Z. G. Wu, Origin of morphotropic phase boundaries in ferroelectrics, Nature 451 (7178), 545 (2008).

${ }^{46}$ C. J. Stringer, R. E. Eitel, T. R. Shrout, C. A. Randall and I. M. Reaney, Phase transition and chemical order in the ferroelectric perovskite $(1-\mathrm{x}) \mathrm{Bi}\left(\mathrm{Mg}_{3 / 4} \mathrm{~W}_{1 / 4}\right) \mathrm{O}_{3}-\mathrm{xPbTiO}_{3}$ solid solution system, J. Appl. Phys. 97, 024101 (2005).

${ }^{47}$ H. Amorín, R. Jiménez, E. Vila, M. Dollé, A. Castro and M. Algueró, Electrical properties of ferroelectric $\mathrm{BiMnO}_{3}-\mathrm{PbTiO}_{3}$ 
under tailored synthesis and ceramic processing, Phase Transit. 86(7), 681 (2013).

${ }^{48}$ M. Algueró, B. Jiménez and L. Pardo, Transition between the relaxor and ferroelectric states for (1-x) $\mathrm{Pb}\left(\mathrm{Mg}_{1 / 3} \mathrm{Nb}_{2 / 3}\right) \mathrm{O}_{3}-\mathrm{X}$ $\mathrm{PbTiO}_{3}$ with $\mathrm{x}=0.2$ and 0.3 polycrystalline aggregates, Appl. Phys. Lett. 87(8), 082910 (2005).

${ }^{49}$ R. Jimenez, B. Jimenez, J. Carreaud, J. M. Kiat, B. Dkhil, J. Holc, M. Kosec and M. Algueró, Transition between the ferroelectric and relaxor states in $0.8 \mathrm{~Pb}\left(\mathrm{Mg}_{1 / 3} \mathrm{Nb}_{2 / 3}\right) \mathrm{O}_{3}-0.2 \mathrm{PbTiO}_{3}$ ceramics, Phys. Rev. B 74(18), 184106 (2006).

${ }^{50} \mathrm{M}$. Algueró, B. Jiménez and L. Pardo, Rayleigh type behavior of the Young's modulus of unpoled ferroelectric ceramics and its dependence on temperature, Appl. Phys. Lett. 83(13), 2641 (2003).

${ }^{51}$ M. Algueró, R. Jiménez, H. Amorín, E. Vila and A. Castro, Low temperature phenomena in ferroic $\mathrm{BiMO}_{3}-\mathrm{PbTiO}_{3}(\mathrm{M}: \mathrm{Mn}$ and Sc), Appl. Phys. Lett. 98(20), 202904 (2011).

${ }^{52} \mathrm{P}$. Baettig, R. Seshadri and N. A. Spaldin, Anti-polarity in ideal $\mathrm{BiMnO}_{3}$, J. Am. Chem. Soc. 129(32), 9854 (2007).

${ }^{53}$ A. A. Belik, S. Ikubo, K. Kodama, N. Igawa, S. Shamoto, M. Maie, T. Nagai, Y. Matsui, S. Y. Stefenovich, B. I. Lazoryak and E. Takayma-Muromachi, $\mathrm{BiScO}_{3}$ :Centrosymmetric $\mathrm{BiMnO}_{3}$-type oxide, J. Am. Chem. Soc. 128(3), 706 (2006).

${ }^{54}$ T. Yokosawa, A. A. Belik, T. Asaka, K. Kimoto, E. TakayamaMuromachi and Y. Matsui, Crystal symmetry of $\mathrm{BiMnO}_{3}$ : Electron diffraction study, Phys. Rev. B 77, 024111 (2008).

${ }^{55}$ V. Goian, S. Kamba, M. Savinov, D. Nuzhnyy, F. Borodavka, P. Vaněk and A. A. Belik, Absence of ferroelectricity in $\mathrm{BiMnO}_{3}$ ceramics, J. Appl. Phys. 112, 074112 (2012).

${ }^{56}$ W. Kaczmarek, Z. Pajak and M. Polomska, Differential thermal analysis of phase transitions in $\left(\mathrm{Bi}_{1-x} \mathrm{La}_{x}\right) \mathrm{FeO}_{3}$ solid solution, Solid State Commun. 17, 807 (1975).

${ }^{57}$ F. Kubel and H. Schmid, Structure of a ferroelectric and ferroelastic monodomain crystal of the perovskite $\mathrm{BiFeO}_{3}$, Acta Crystallogr. Sect. B 46, 698 (1990).

${ }^{58}$ D. Lebeugle, D. Colson, A. Forget and M. Viret, Very large spontaneous polarization on $\mathrm{BiFeO}_{3}$ single crystals at room temperature and its evolution under cycling fields, Appl. Phys. Lett. 91, 022907 (2007).

${ }^{59}$ J. M. Moreau, C. Michel, R. Gerson and W. J. James, Ferroelectric $\mathrm{BiFeO}_{3} \mathrm{X}$-ray and neutron diffraction study, J. Phys. Chem. Solids 32, 1315 (1971).

${ }^{60} \mathrm{C}$. Ederer and N. A. Spaldin, Weak ferromagnetism and magnetoelectric coupling in bismuth ferrite, Phys. Rev. B 71, 0604401(R) (2005).

${ }^{61}$ R. T. Smith, G. D. Achenbach, R. Gerson and W. J. James, Dielectric properties of solid solutions of $\mathrm{BiFeO}_{3}$ with $\mathrm{Pb}(\mathrm{Ti}, \mathrm{Zr})$ $\mathrm{O}_{3}$ at high temperature and high frequency, J. Appl. Phys. 39, 70 (1968).

${ }^{62}$ D. I. Woodward, I. M. Reaney, R. E. Eitel and C. A. Randall, Crystal and domain structure of the $\mathrm{BiFeO}_{3}-\mathrm{PbTiO}_{3}$ solid solution, J. Appl. Phys. 94, 3313 (2003).

${ }^{63}$ W. M. Zhu, H. Y. Guo and Z. G. Ye, Structural and magnetic characterization of multiferroic $\left(\mathrm{BiFeO}_{3}\right)_{1-x}\left(\mathrm{PbTiO}_{3}\right)_{x}$ solid solutions, Phys. Rev. B 78, 014401 (2008).

${ }^{64} \mathrm{~S}$. Bhattacharje, S. Tripathi and D. Pandey, Morphotropic phase boundary in (1-x) $\mathrm{BiFeO}_{3}-\mathrm{xPbTiO}_{3}$ : Coexistence region and unusually large tetragonality, Appl. Phys. Lett. 91, 042903 (2007).
${ }^{65}$ C. Correas, T. Hungría and A. Castro, Mechanosynthesis of the whole $\mathrm{xBiFeO}_{3}-(1-\mathrm{x}) \mathrm{PbTiO}_{3}$ multiferroic system: Structural characterization and study of phase transitions, J. Mater. Chem. 21, 3125 (2011).

${ }^{66} \mathrm{~S}$. Bhattacharje and D. Pandey, Stability of the various crystallographic phases of the multiferroic $(1-\mathrm{x}) \mathrm{BiFeO}_{3}-\mathrm{xPbTiO}_{3}$ system as a function of composition and temperature, J. Appl. Phys. 107, 124112 (2010).

${ }^{67}$ T. P. Comyn, T. Stevenson, M. A. Al-Jawad, S. L. Turner, R. Smith, A. J. Bell and R. Cywinsky, High temperature neutron diffraction studies of $0.9 \mathrm{BiFeO}_{3}-0.1 \mathrm{PbTiO}_{3}$, J. Appl. Phys. 105, 094108 (2009).

${ }^{68}$ S. Bhattacharje, A. Senyshyn, P. S. R. Krishna, H. Fuess and D. Pandey, Simultaneous changes of nuclear and magnetic structures across the morphotropic phase boundary in (1-x) $\mathrm{BiFeO}_{3}-\mathrm{xPTiO}_{3}$, Appl. Phys. Lett. 97, 262506 (2010).

${ }^{69}$ T. Comyn, T. Stevenson, M. Al-Jawad, S. L. Turner, R. I. Smith, W. G. Marshall, A. J. Bell and R. Cywinski, Phase-specific magnetic ordering in $\mathrm{BiFeO}_{3}-\mathrm{PbTiO}_{3}$, Appl. Phys. Lett. 93, 232901 (2010).

${ }^{70}$ S. Bhattacharje, A. Senyshyn, H. Fuess and D. Pandey, Morintype spin-reorientation below the Neel transition in the monoclinic compositions of $(1-\mathrm{x}) \mathrm{BiFeO}_{3}-\mathrm{xPbTiO}_{3}(\mathrm{x}=0.25$ and 0.27$)$ : $\mathrm{A}$ combined dc magnetization and x-ray and neutron powder diffraction study, Phys. Rev. B 87, 054417 (2013).

${ }^{71}$ H. Amorín, C. Correas, C. M. Fernández-Posada, O. Peña, A. Castro and M. Algueró, Multiferroism and enhancement of materials properties across the morphotropic phase boundary of $\mathrm{BiFeO}_{3}-\mathrm{PbTiO}_{3}$, J. Appl. Phys. 115, 104104 (2014).

${ }^{72}$ H. Amorín, C. Correas, P. Ramos, T. Hungría, A. Castro and M. Algueró, Very high remnant polarization and phase-change electromechanical response of $\mathrm{BiFeO}_{3}-\mathrm{PbTiO}_{3}$ at the multiferroic morphotropic phase boundary, Appl. Phys. Lett. 101, 172908 (2012).

${ }^{73}$ T. P. Comyn, S. P. McBride and A. J. Bell, Processing and electrical properties of $\mathrm{BiFeO}_{3}-\mathrm{PbTiO}_{3}$ ceramics, Mater. Lett. 58, 3844 (2004).

${ }^{74}$ R. E. Eitel, S. J. Zhang, T. R. Shrout, C. A. Randall and I. Levin, Phase diagram of the perovskite system $(1-\mathrm{x}) \mathrm{BiScO}_{3}-\mathrm{PbTiO}_{3}$, J. Appl. Phys. 96, 2828 (2004).

${ }^{75}$ R. Theissmann, L. A. Schmitt, J. Kling, R. Schierholz, K. A. Schönau, H. Fuess, M. Knapp, H. Kungl and M. J. Hoffmann, Nanodomains in morphotropic lead zirconate titanate ceramics: On the origin of the strong piezoelectric effect, J. Appl. Phys. 102, 024111 (2007).

${ }^{76}$ Q. M. Zhang, H. Wang, N. Kim and L. E. Cross, Direct evaluation of domain-wall and intrinsic contributions to the dielectric and piezoelectric response and their temperature dependence on lead zirconate-titanate ceramics, J. Appl. Phys. 75, 454 (1994).

${ }^{77}$ S. Bhattacharjee, V. Pandey, R. K. Kotnala and D. Pandey, Unambiguous evidence for magnetoelectric coupling of multiferroic origin in $0.73 \mathrm{BiFeO}_{3}-0.27 \mathrm{PbTiO}_{3}$, Appl. Phys. Lett. 94, 012906 (2009).

${ }^{78}$ C. M. Fernández-Posada, H. Amorín, C. Correas, O. Peña, M. Algueró and A. Castro, Mechanosynthesis and multiferroic properties of the $\mathrm{BiFeO}_{3}-\mathrm{BiMnO}_{3}-\mathrm{PbTiO}_{3}$ ternary system along the morphotropic phase boundary, J. Mater. Chem. C 3, 2255 (2015).

${ }^{79}$ C. M. Fernández-Posada, A. Castro, J. M. Kiat, F. Porcher, O. Peña, M. Algueró and H. Amorín, in preparation. 